\title{
Traitement orthopédique des classes III par stimulation de la croissance du maxillaire
}

\section{Orthopaedic treatment of the classes III by stimulation of the growth of the jaw}

\section{Résumé}

\section{MOTS-CLEFS :}

- Classe III, hypoplasie maxillaire, prognathie mandibulaire, surélévations molaires, traitement fonctionnels précoces

\section{KEYWORDS:}

- Classe III, hypoplasie maxillary, prognathie mandibular, extra height molars, treatment functional early

AOS $n^{\circ} 287-2018$
Le manque de développement du maxillaire génère un hyperdéveloppement mandibulaire dans les 3 plans de l'espace.

Cette insuffisance de croissance est tout à fait rattrapable et majorable et d'autant plus que l'enfant est jeune avec de la croissance à venir.

L'utilisation d'appareillages fonctionnels permet aux dents maxillaires de recevoir les stimuli masticatoires, et au maxillaire de se développer dans les 3 plans de l'espace en même temps que la mandibule se repositionne.
Abstract

The lack of development of the jaw generates a mandibular hyperdevelopment in 3 plans of the space.

This insufficiency of growth is completely recoverable and majorable and especially as the child is young with the growth to come.

The use of functional equipments allows the maxillary teeth to receive stimuli masticatoires, and in the jaw to develop in 3 plans of the space at the same time as the mandible repositions.

Carine BEN YOUNES-UZAN,
Chirurgien-Dentiste
Spécialiste Qualifié Orthopédie Dento-Faciale, Paris
Ancien attaché de consultation à l'hôpital Robert Debré
Membre du bureau de la SBR, Paris
c.benyounes@wanadoo.fr

\section{INTRODUCTION}

Les traitements orthopédiques lourds et la chirurgie sont souvent proposés comme seule issue thérapeutique à la résolution des prognathies mandibulaires et des hypoplasies maxillaires. Les articulés inversés sont détectables très précocement dans la petite enfance dès la denture lactéale.
De nombreux auteurs dont Mc Namara et Miyama [1] ont montré qu'en l'absence de traitement, les rétrusions maxillaires ne s'améliorent pas avec le temps, tandis que la protrusion mandibulaire augmente. L'ensemble conduit toujours à l'aggravation de la dysharmonie squelettique de départ. 
En général, les parents se rendent compte assez tôt qu'il y a un problème au niveau des mâchoires de leur enfant et dès ce moment, il faut intervenir.

Une perte de la dimension verticale d'occlusion par édentation bilatérale postérieure génère des rapports intermaxillaires de classe III, comme c'est souvent le cas chez le vieillard qui n'a pourtant plus de croissance.

Chez l'enfant en croissance, l'augmentation de la dimension verticale d'occlusion, par une surélévation sur les dents postérieures, va permettre de freiner l'avancée mandibulaire et de promouvoir la croissance maxillaire.

\section{HISTORIQUE}

L'école française des fonctionnalistes, au départ essentiellement constituée de médecins stomatologues, s'est attachée depuis Robin à traiter d'un point de vue médical les dysharmonies squelettiques.

Dans cette lignée médicale, le Professeur Deffez a dirigé pendant de nombreuses années le service de stomatologie pédiatrique des hôpitaux Bretonneau, puis Robert Debré. Les traitements d'Orthopédie Dento-Faciale y étaient réalisés sans bague, à l'aide uniquement d'appareils amovibles fonctionnels [2-4]. Le rôle de la langue et des tissus mous étant essentiel pour le façonnage des structures osseuses et dentaires, ces traitements faisaient partie d'une approche médicale pluridisciplinaire globale : orthophonique, ORL, psychologique, ostéopathique...

Ces traitements fonctionnels sans multiattache donnent d'excellents résultats avant la denture définitive.

S'appuyant sur les travaux de Lepoivre [5], Deffez [2-4] puis Fellus [6] ont traité les hypoplasies maxillaires en utilisant uniquement des dispositifs amovibles accroissant la dimension verticale d'occlusion et ont expliqué leur action d'un point de vue neuro-musculaire.

Delaire [9-10] et Planas [11] ont aussi expliqué l'architectonique et la dynamique cranio-faciale ainsi que le développement fonctionnel de la face en ajoutant le rôle de la frappe occlusale et de la mastication.

Un antagonisme entre les dents et la langue fait le pont entre les différentes approches fonctionnelles.

La langue recule pour ne pas "se faire manger " quand se met en route la dynamique masticatoire, ou persiste entre les dents du fait de la succion-déglutition ou de la respiration buccale [7].
L'association des techniques mécaniques aux concepts fonctionnels permet d'aller plus loin dans les possibilités thérapeutiques [8].

\section{PRINCIPE DU TRAITEMENT}

Étant donné que nous traitons des enfants en croissance et qu'aucun organe n'a atteint sa taille adulte, il est cohérent de promouvoir la croissance de la structure osseuse proportionnellement en retard par l'intermédiaire des procès alvéolo-dentaires sur lesquels nous prenons appui.

Un appareillage amovible porté constamment augmente la dimension verticale d'occlusion, ceci va stimuler la croissance du maxillaire et réorienter la croissance mandibulaire.

Le recouvrement physiologique maxillo-mandibulaire dans les 3 plans de l'espace va permettre lors de la mastication, le frottement de toutes des dents supérieures par les dents inférieures.

En cas d'articulé croisé ou d'infraclusion les dents maxillaires ne recouvrent pas ou incorrectement leurs antagonistes, ces dents en mauvaise position ainsi que l'os qui les porte ne perçoivent pas les stimuli venant du frottement des dents antagonistes; tandis que la mandibule n'est plus contenue.

Quand la mandibule n'est pas retenue par le maxillaire, sa croissance n'est pas maîtrisée et elle peut partir dans les 3 plans de l'espace : vers l'avant-prognathie mandibulaire, le côté-exoclusion mandibulaire, où le bas-hyperdivergence squelettique.

La base du traitement est la majoration de la dimension verticale d'occlusion fonctionnelle et la mise en fonction de toutes les dents maxillaires; en denture lactéale, un appareil amovible seul peut suffire, mais dès l'apparition des dents définitives, nous mettrons en place conjointement des attaches collées pour aligner les dents et amplifier les effets désirés.

Nous utilisons une plaque de Hawley munie d'un vérin d'activation et de surélévations molaires. Le facteur clé est l'épaisseur des cales molaires, d'une hauteur importante, toujours supérieure à l'espace libre d'inocclusion.

La plaque en résine va être en contact avec toutes les dents maxillaires, la frappe occlusale et les frottements masticatoires pourront être transmis à toutes les dents supérieures, y compris celles qui étaient en articulé croisé ou en inocclusion.

L'appareil amovible mono-maxillaire doit être porté continuellement même lors des repas ce qui supprime toute mémoire proprioceptive de l'articulé croisé 

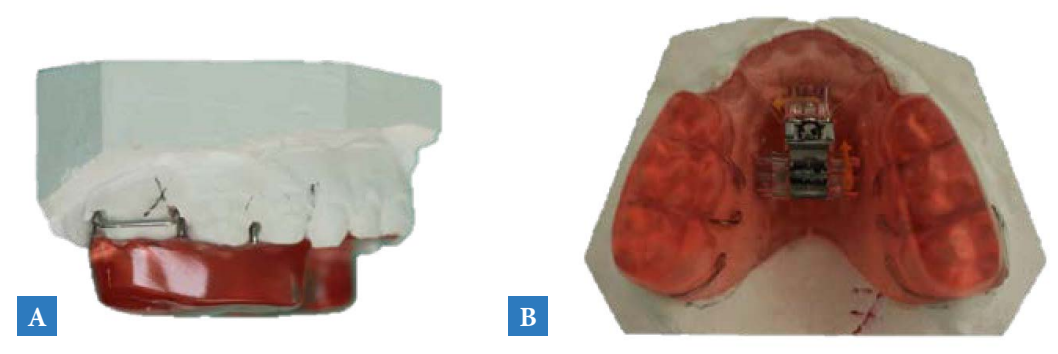

$\succ$ Fig. $1 \mathrm{~A}$ et $\mathrm{B}$.

et empêche tout contact prématuré à l'origine des déviations du chemin de fermeture. Il n'y a plus ni proglissement, ni latéro-déviation.

La surélévation molaire stimule la croissance maxillaire dans les 3 sens de l'espace.

\section{Dans le sens antéro-postérieur}

Une inversion d'articulé empêche toute possibilité d'expansion naturelle des dents maxillaires, car à chaque fermeture, elles subissent une force concentrique s'opposant à l'épanouissement de l'arcade. L'épaisseur de résine interposée entre les dents va libérer les dents supérieures de l'occlusion pathologique ;

La hauteur importante des surélévations modifie l'orientation des forces de la mastication :

De verticales, elles deviennent obliques avec une composante postéro-antérieure qui pousse le maxillaire vers l'avant, comme le masque de Delaire qui le tire. La résine étant au contact de la face linguale des incisives supérieures, les sollicitations fonctionnelles de la frappe occlusale (Deffez) [2-4] et du frottement masticatoire (Planas) [11] sont transmises à ces dents, donnent l'énergie et entraînent l'expansion de cette arcade.

La hauteur efficace, pour obtenir l'antériorisation du prémaxillaire, est obtenue quand la mandibule est abaissée donc reculée suffisamment pour simuler des rapports incisifs normaux dans le sens antéro-postérieur. Plus on veut un effet d'avancée maxillaire, plus la hauteur de résine doit être importante.

L'avancée et la descente du condyle dans la cavité glénoïde modifient l'orientation des ligaments et des muscles, en particulier des ptérygoïdiens latéraux. Leur axe est transformé, initialement horizontal, il devient oblique; et en se verticalisant, les muscles perdent alors une partie de leur action propulsive qui agissait sur la mandibule.

Les tissus mous voient leur action modifiée.

La langue en général très puissante chez ces patients peut exercer son action centrifuge sur les incisives supérieures, qui ne sont plus retenues par l'occlusion. Les cales latérales de résine vont lui accroître le volume disponible à l'intérieur des arcades dentaires, qui subiront la poussée de celle-ci.

Comme leur épaisseur est supérieure à l'espace libre d'inocclusion, l'interposition linguale latérale entre les arcades est empêchée et la proprioception dentaire est stimulée car les dents postérieures retrouvent un contact par l'intermédiaire des plans molaires.

Le palais en résine peut, en venant au contact de la langue, recréer une proprioception tactile du dôme lingual, entraînant l'élévation de celle-ci.

L'orbiculaire inférieur n'exercera plus de contraintes sur les incisives supérieures libérées, mais plutôt sur les dents mandibulaires.

L'orbiculaire supérieur moins tonique, offrira moins de résistance à la poussée linguale sur les incisives maxillaires.

\section{Dans le sens transversal}

Comme les dents latérales supérieures sont englobées dans la résine, les activations du vérin d'expansion n'engendrent pas de version coronaire de ces dents mais un mouvement en gression. La suture intermaxillaire est activement sollicitée et provoque un déplacement homothétique en expansion des procès alvéolo-dentaires, donnant la place nécessaire aux dents définitives qu'il suffit d'aligner.

\section{Dans le sens vertical}

La surélévation molaire contribue à fermer le sens vertical en ingressant les secteurs postérieurs recouvert de résine et laissant l'égression spontanée des dents antérieures s'exprimer. Le meulage des contacts canins qui vont s'obtenir avant le recouvrement incisif, majore cet effet. Les plans molaires fonctionnent selon le même principe que les surélévations rétro-incisives. La résine appliquée contre toutes les dents, donne des stimuli proprioceptifs aux dents antérieures, qui peuvent en être dépourvues du fait d'un articulé croisé ou d'une infraclusion. 


\section{PRÉSENTATION DE CAS CLINIQUES}

\section{Cas clinique $n^{\circ} 1$ (Fig. 2 à 16)}

Petite fille de 5 ans 3 mois, elle présente un déficit marqué de l'étage moyen de la face. Sa lèvre supérieure, très en retrait, a son bord rouge peu visible.

Sa lèvre inférieure est éversée, son sourire découvre ses incisives mandibulaires (Fig. 2A à C).

Elle est en denture lactéale, ses dents de 6 ans ne sont pas encore évoluées (Fig. 3A à C).

Sur la radiographie panoramique, comme dans toutes les hypoplasies maxillaires, on note un manque de place prévisible pour les dents définitives à l'arcade maxillaire, alors qu'elles sont bien espacées à l'arcade mandibulaire hyper développée (Fig. 4).

La téléradiographie met en évidence une classe III aggravée par la proalvéolie des incisives inférieures (Fig. 5).

Nous avons utilisé un appareil à surélévations molaires et très rapidement, le maxillaire s'est avancé. La hauteur efficace pour franchir l'occlusion a été obtenue en 2 temps par ajout de résine au fauteuil. Quand le surplomb incisif est obtenu, la hauteur des plans est progressivement diminuée en maintenant les incisives inférieures par un élastique en port nocturne (Fig. 6A à I).
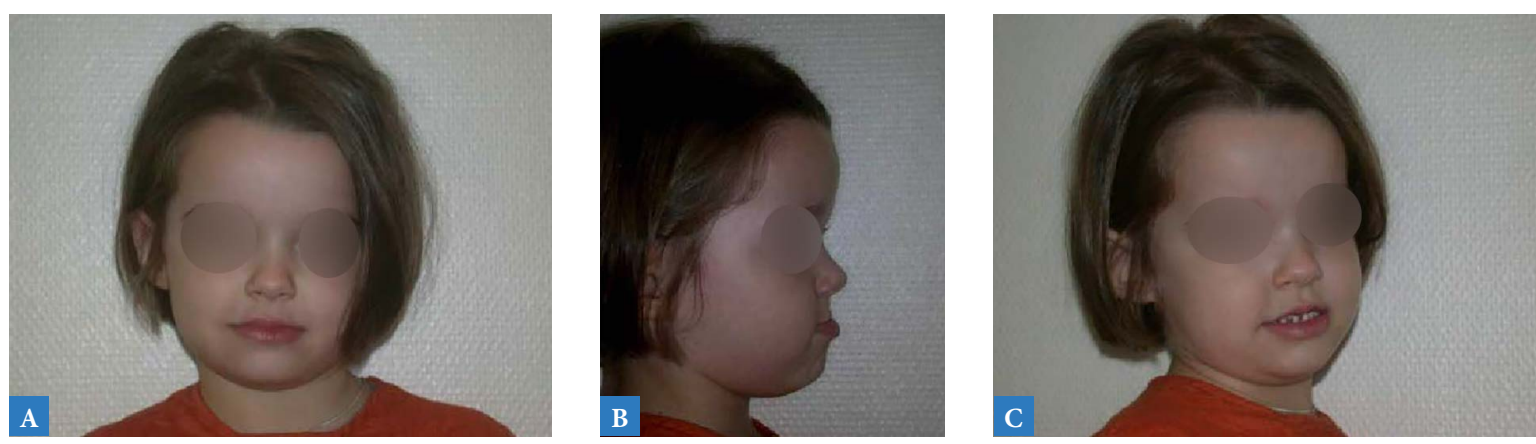

$\triangle$ Fig. 2A à C.
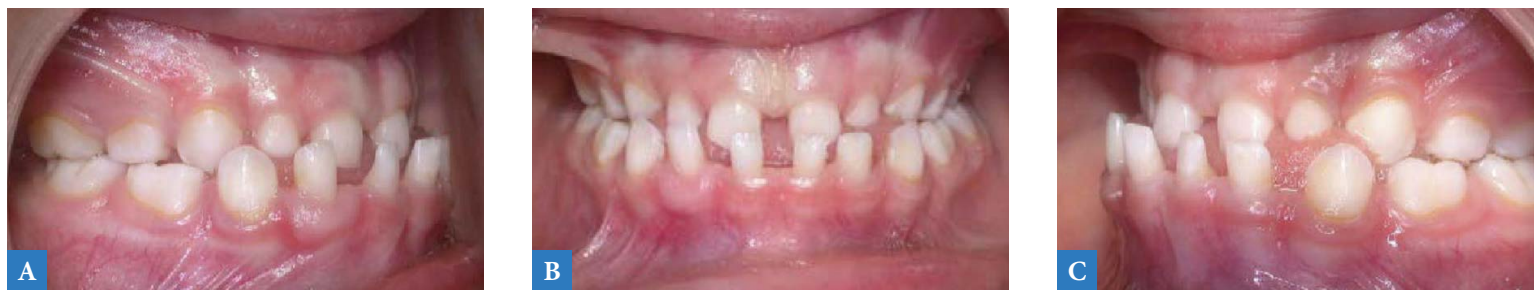

Fig. 3A à C
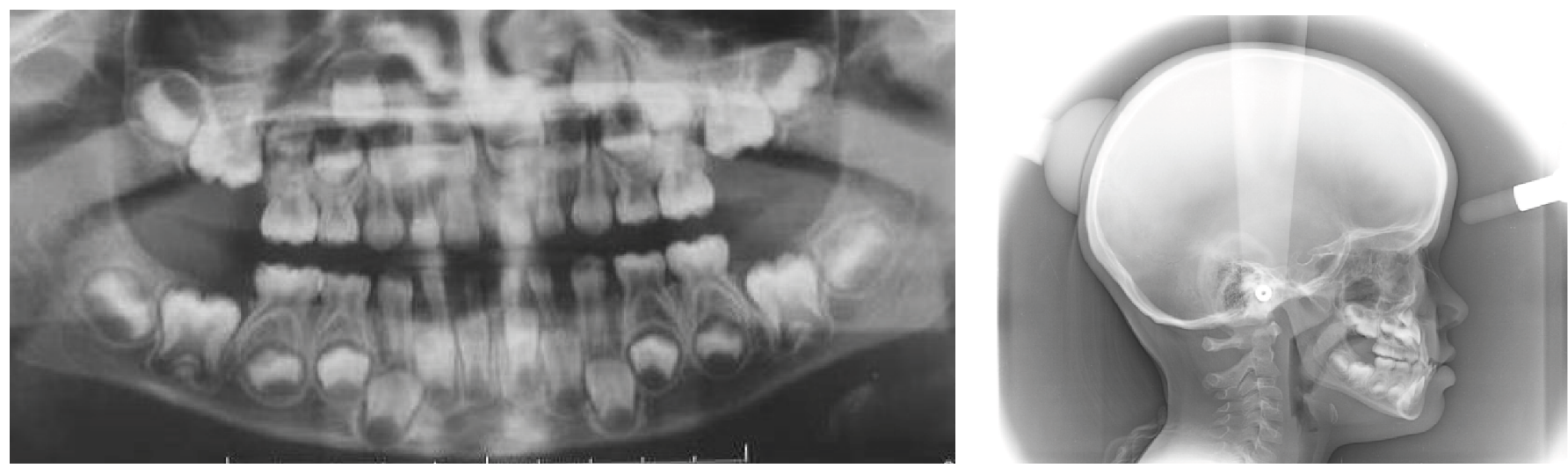

$\triangle$ Fig. 4.

Fig. 5. 

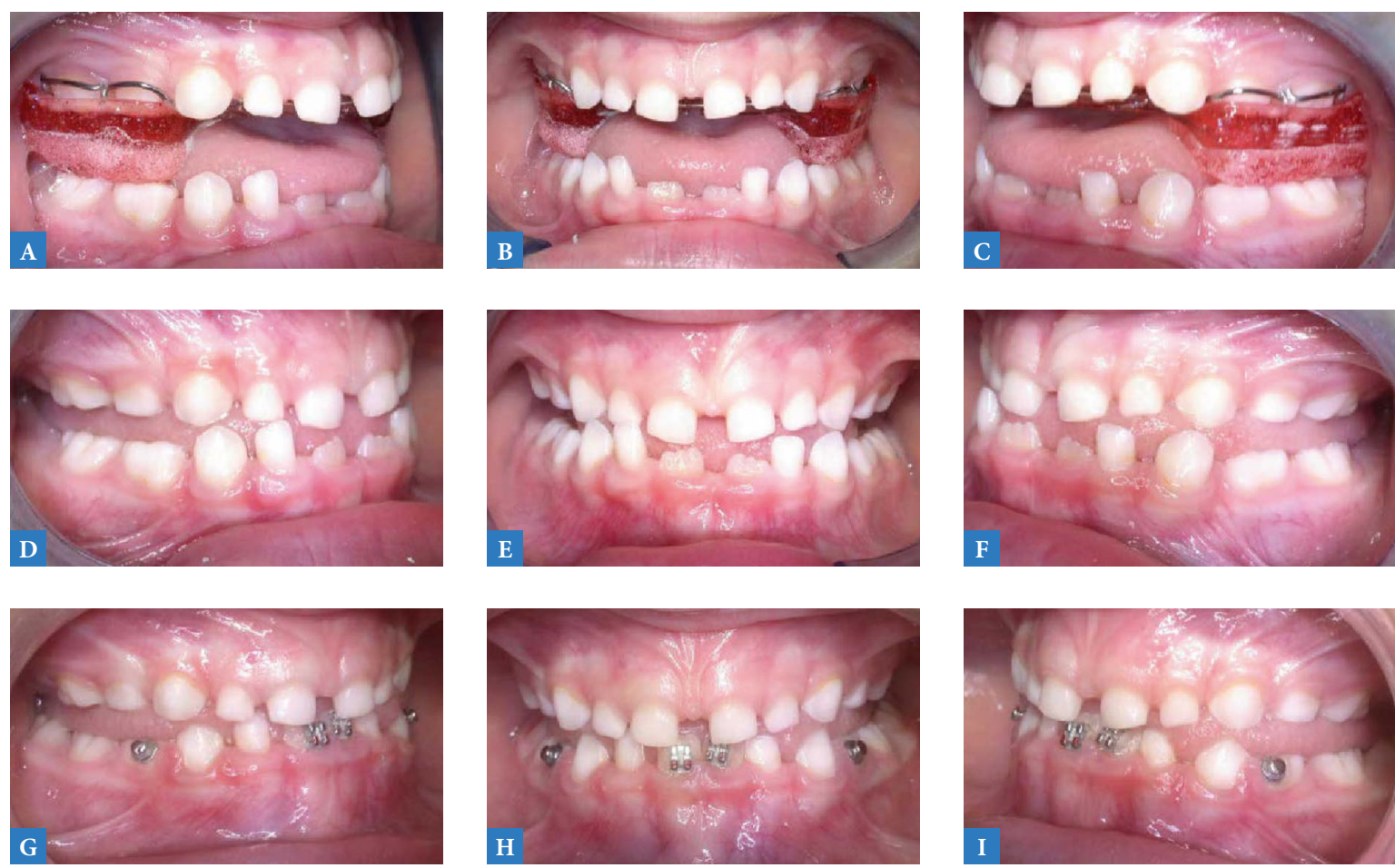

$\triangle$ Fig. 6A à I.
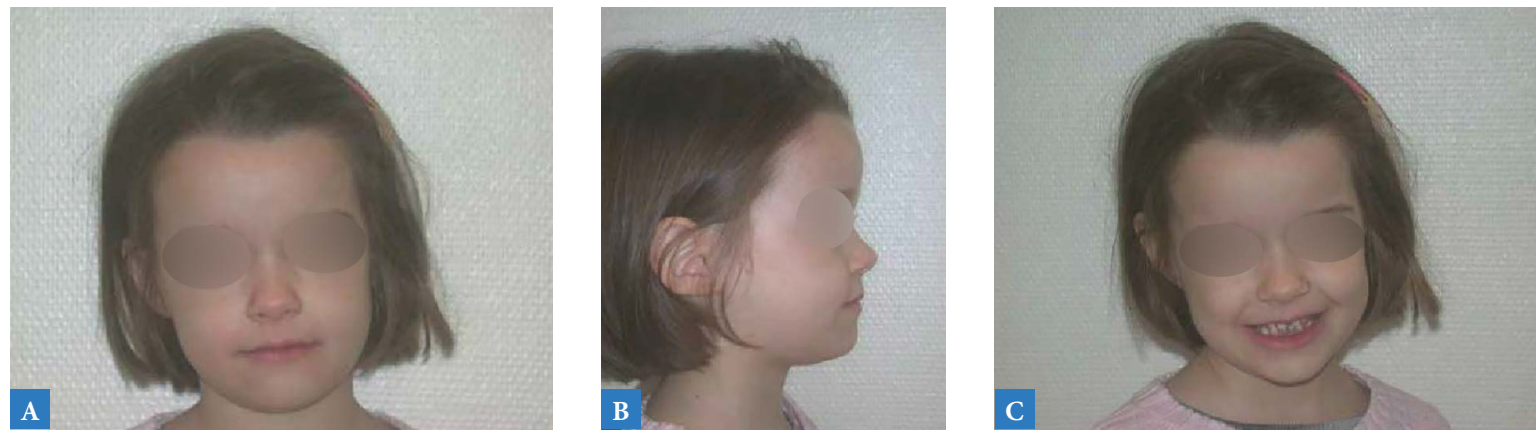

Fig. 7A à C.
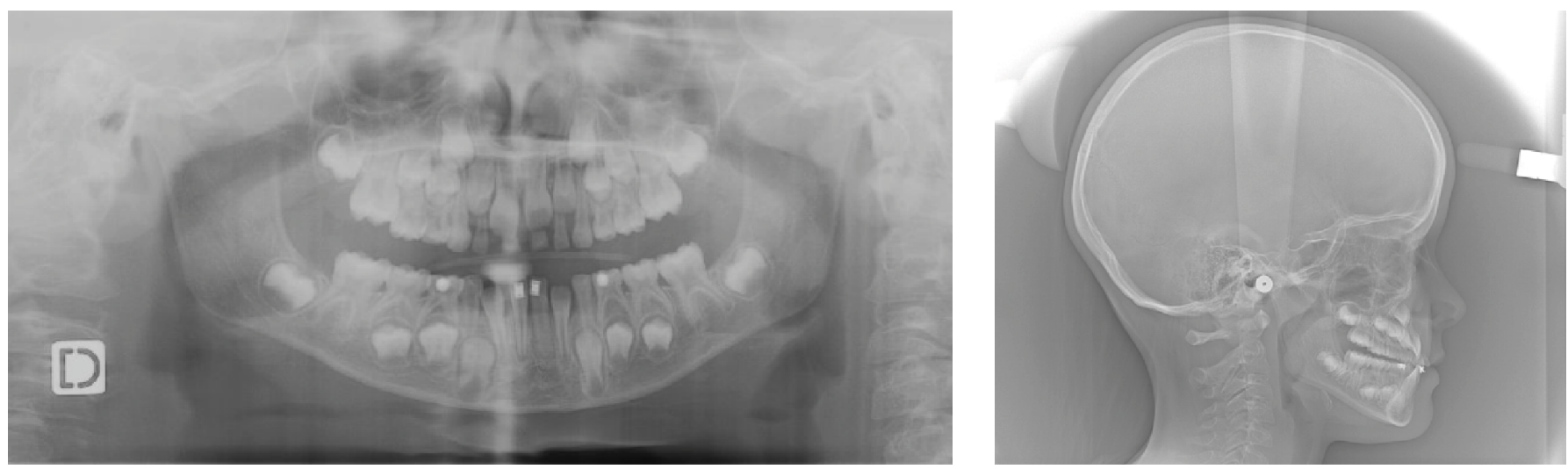

$\triangle$ Fig. 8.

$\triangle$ Fig. 9. 


\section{Superpositions}
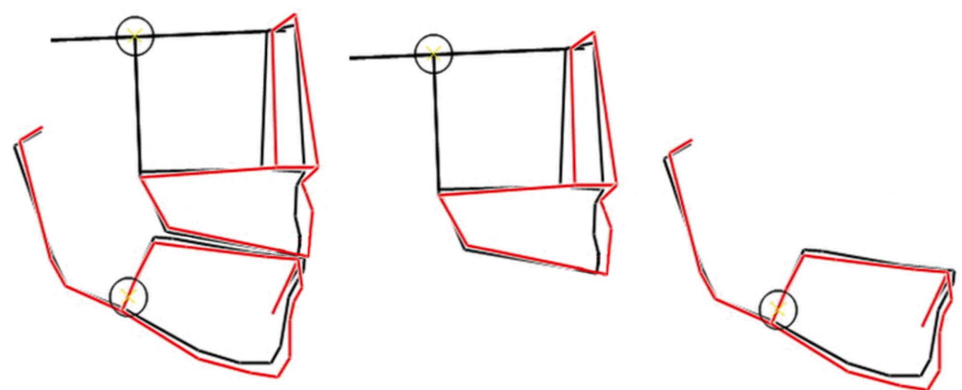

$\triangle$ Fig. 10.

\section{Discussion}

Les superpositions montrent une croissance maxillaire marquée, tandis que la mandibule s'est maintenue. La bascule vers l'avant des piliers antérieurs de la face, autour de la suture fronto-maxillaire, a conduit à l'antériorisation du prémaxillaire.

Son visage s'est harmonisé, l'amélioration concerne tout l'étage moyen de la face : ses lèvres se situent sur le même plan vertical et ont un relief équilibré. L'angle cervico-mentonnier est plus marqué avec disparition $\mathrm{du}$ double menton correspondant une ascension $\mathrm{du}$ corps lingual, le dos de la langue est maintenant au palais, et l'élévation de l'os hyoïde est visible sur la téléradiographie.

La patiente sourit avec les incisives maxillaires (Fig. 11 à 14).

L'examen de son visage, son occlusion et ses radiographies 5 ans après la fin du traitement, avant de réaliser la finition multiattache, les résultats acquis se sont maintenus.

Le traitement multiattache a permis de fermer les diastèmes et de rétablir l'occlusion canine (Fig. 15 à 16).

Fig. $11 \mathrm{~A}$ à C.
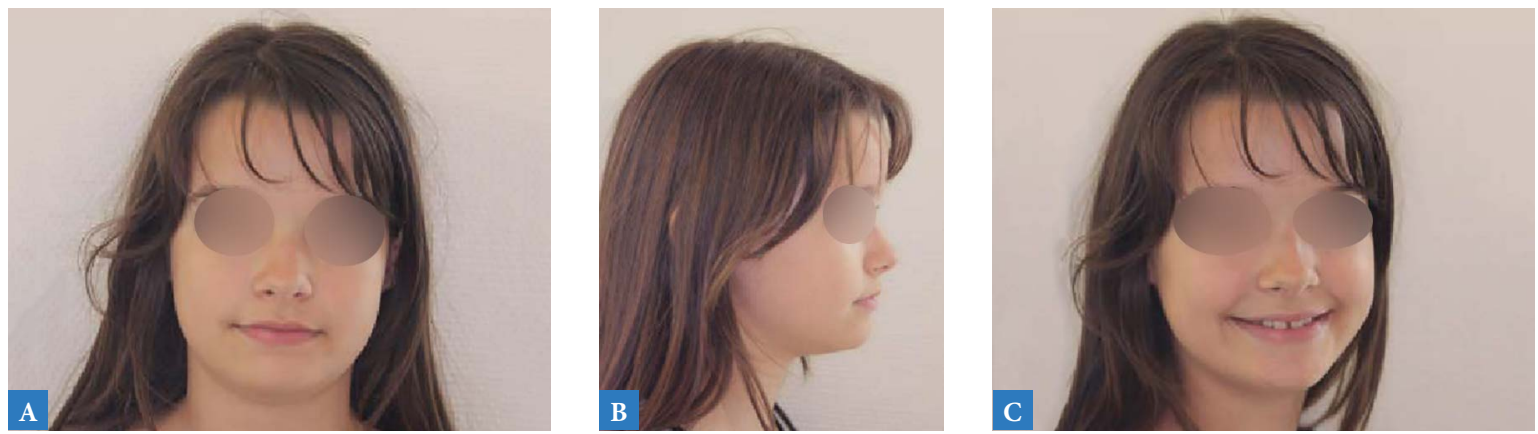

Fig. $12 \mathrm{~A}$ à $\mathrm{C}$.
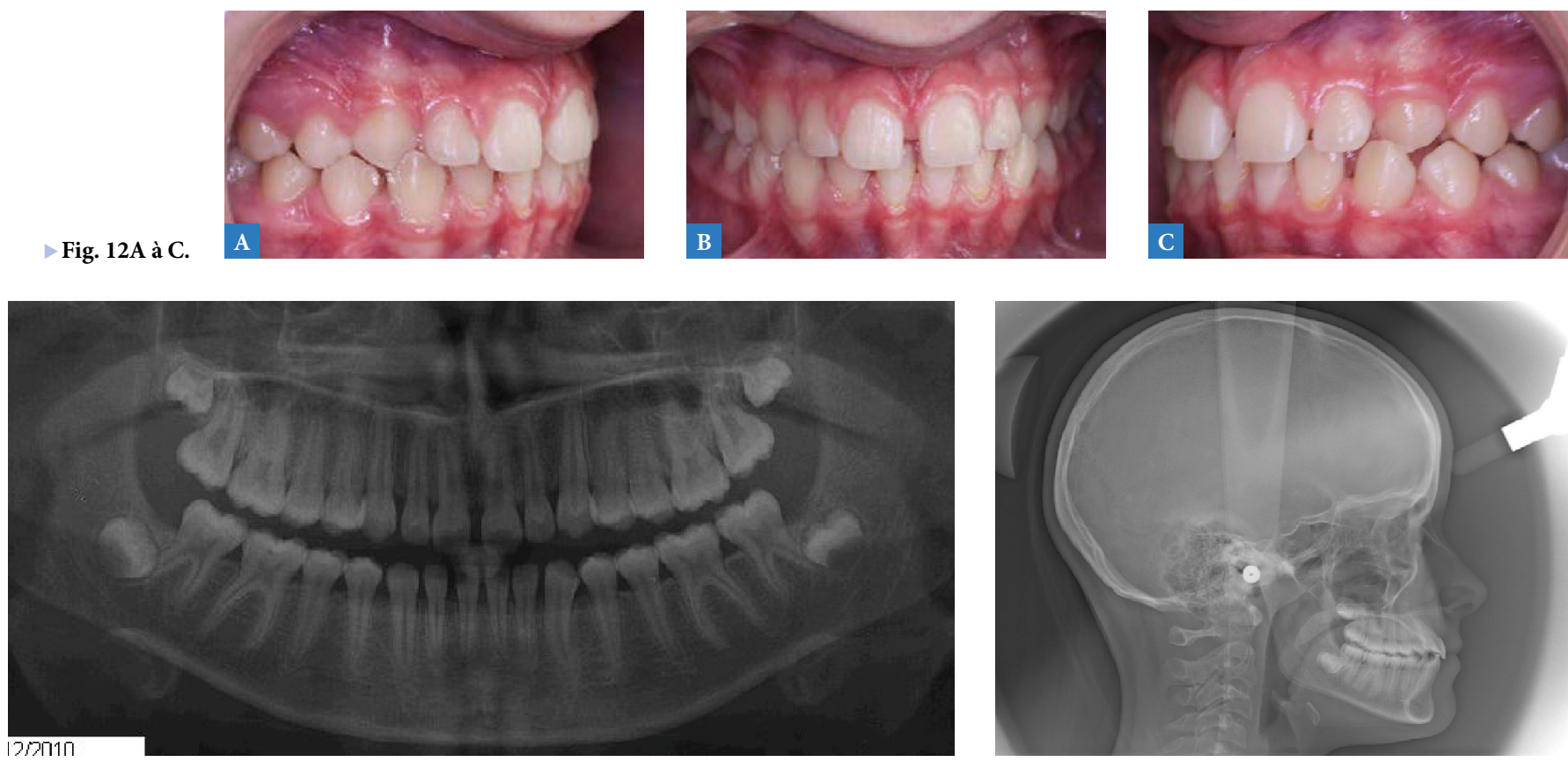

$\triangle$ Fig. 13. 

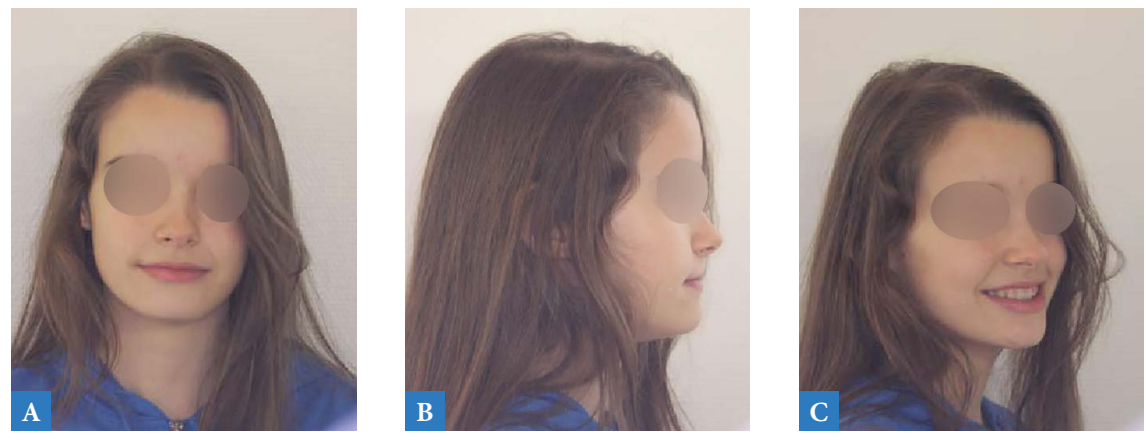

$\triangle$ Fig. $15 \mathrm{~A}$ à $\mathrm{C}$.
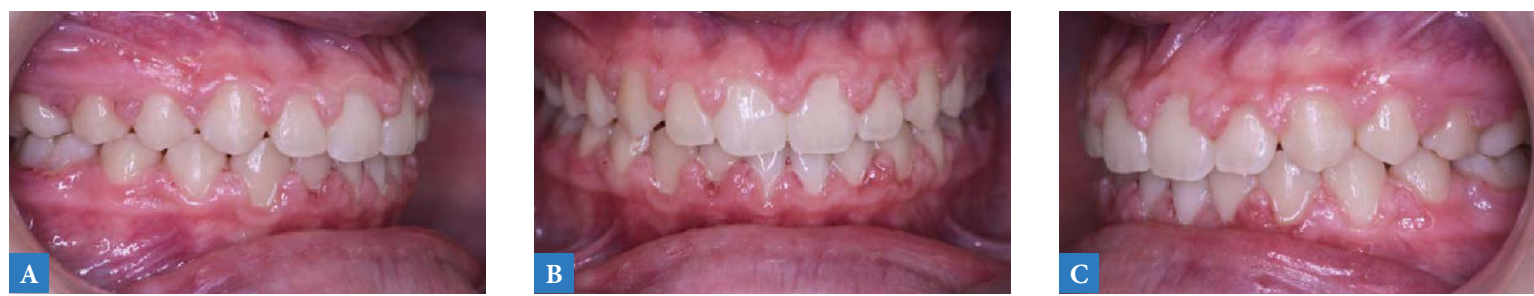

$\triangle$ Fig. 16A à C.

\section{Cas clinique $n^{\circ} 2$ (Fig. 17 à 26)}

Une petite fille de 6 ans 7 mois consulte pour une prognathie mandibulaire.

L'examen de son visage montre une insuffisance de l'étage moyen de la face avec un relief maxillo-malaire trop faible si ce n'est au niveau de la boule graisseuse de Bichat.

Elle a les yeux cernés, sa lèvre inférieure a beaucoup moins de relief que la supérieure.
Son sourire comme dans tous ces cas découvre ses incisives inférieures et donne un aspect de rictus.

La photo de profil masque toujours la déformation par rapport à la téléradiographie de profil car les patients ne sont pas en occlusion, mais au repos avec le plus souvent une interposition de la langue.

Sur la panoramique on voit que toutes les dents sont présentes aux 2 arcades à l'exception des dents de
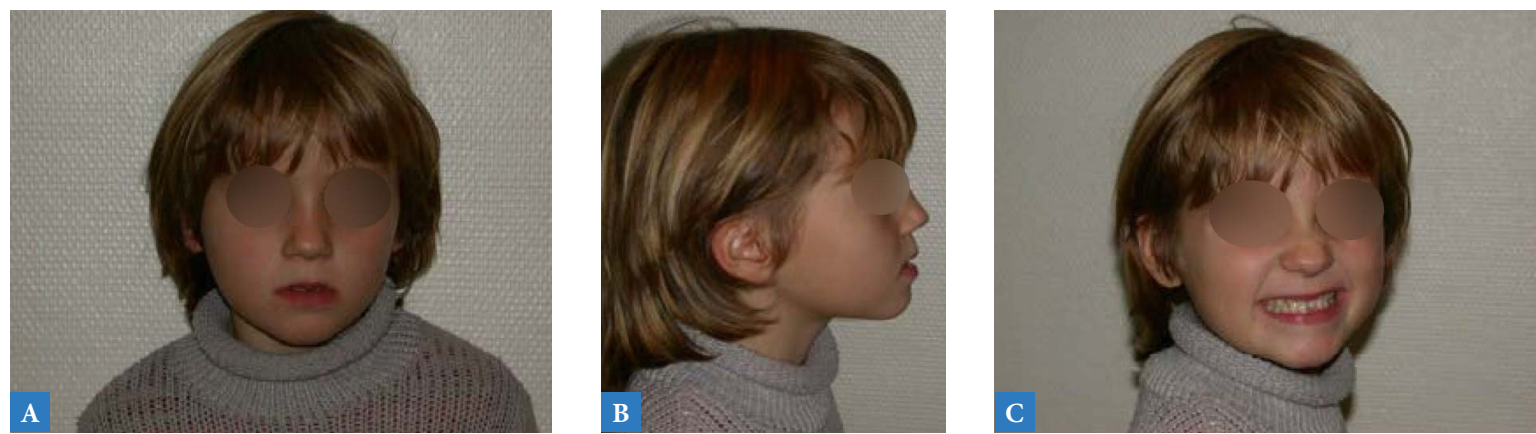

$\triangle$ Fig. 17A à C.
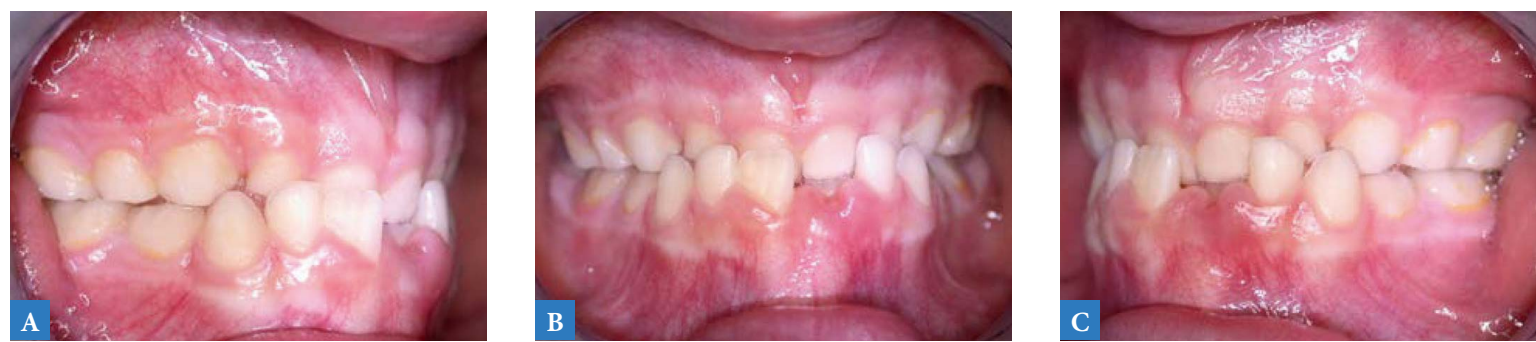

$\triangle$ Fig. 18A à C. 


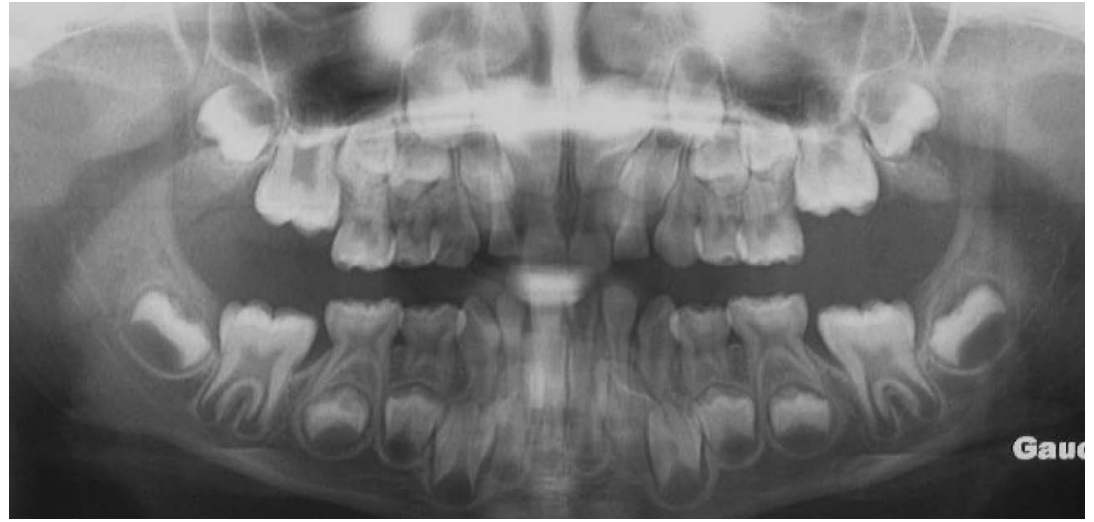

Fig. 19.

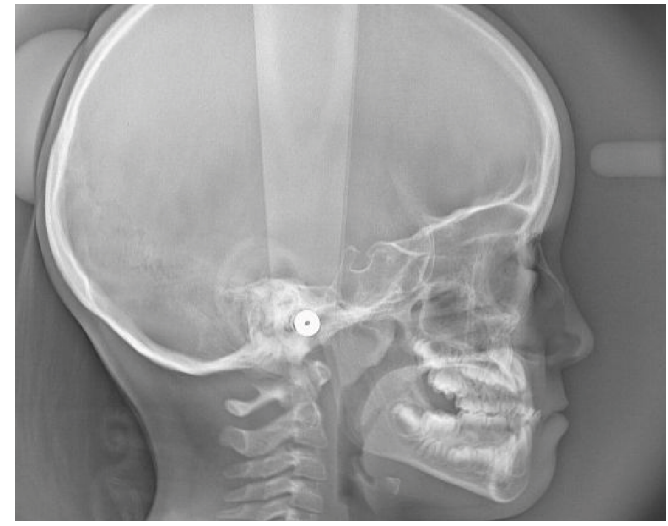

$\triangle$ Fig. 20. sagesse. On présage un manque de place pour les dents définitives, plus marqué à l'arcade supérieure, avec une rotation intra-osseuse des incisives centrales.

La téléradiographie de profil met en évidence la classe III mésodivergente.

Étant donné que nous suivons des enfants en croissance, qu'aucune pièce osseuse n'a atteint sa taille définitive, nous choisissons de prendre en charge la structure en retard qui est ici le maxillaire, pour majorer sa croissance.
Le traitement est débuté par une surélévation molaire, qui doit rester en place à la chute des incisives centrales de lait pour attendre l'éruption des 11 et 21 . La présence des incisives est indispensable pour former un rempart bloquant la mandibule.

C'est pourquoi un recouvrement important même inversé est un pronostic très favorable.

Dès l'éruption des incisives des brackets corrigeront leur rotation mésio-palatine, qui donnait une interférence défavorable.
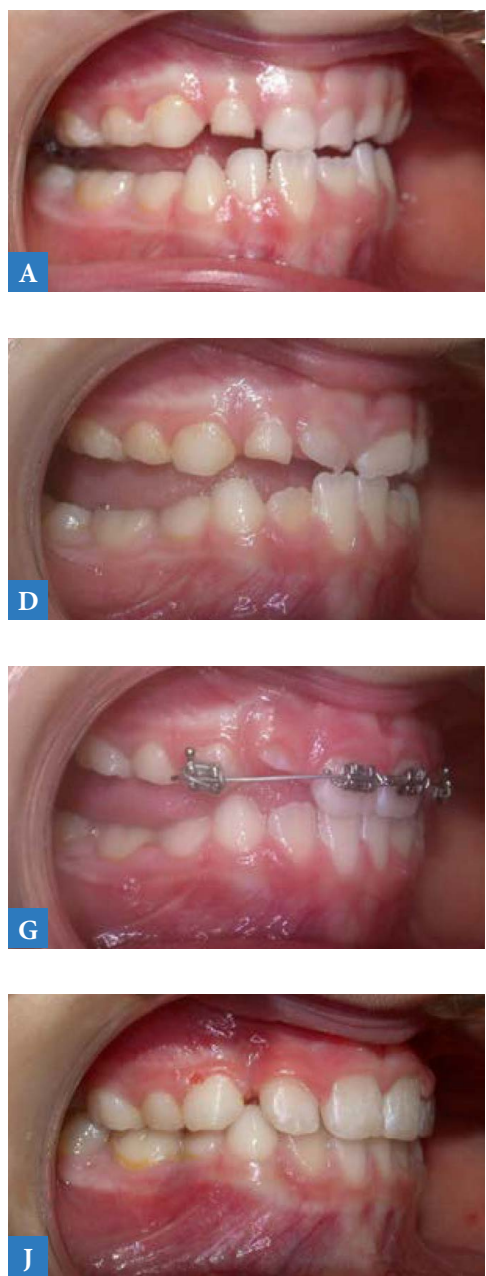

$\triangle$ Fig. $21 \mathrm{~A}$ à $\mathrm{L}$.
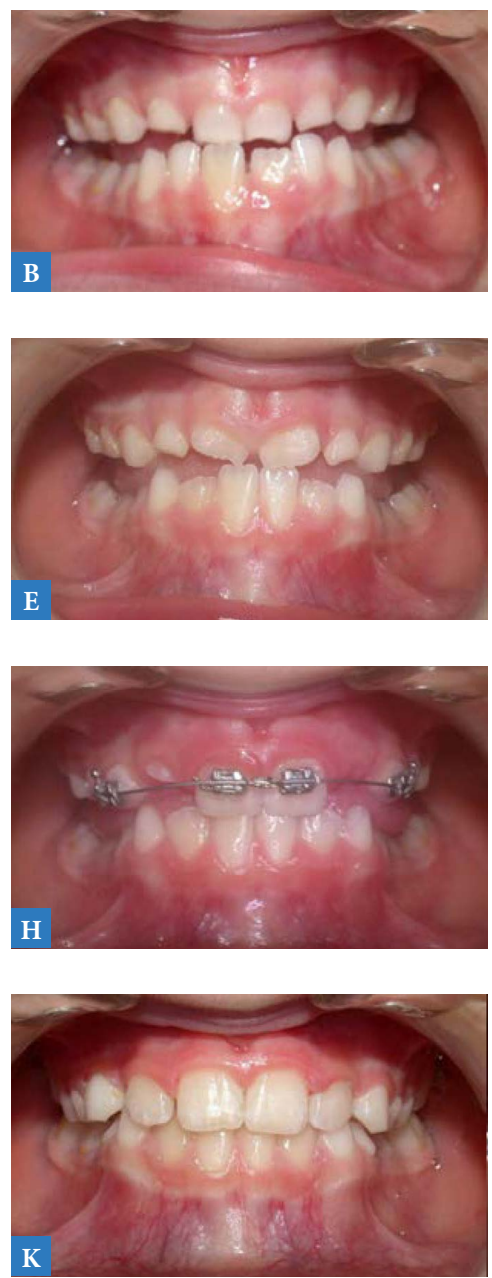
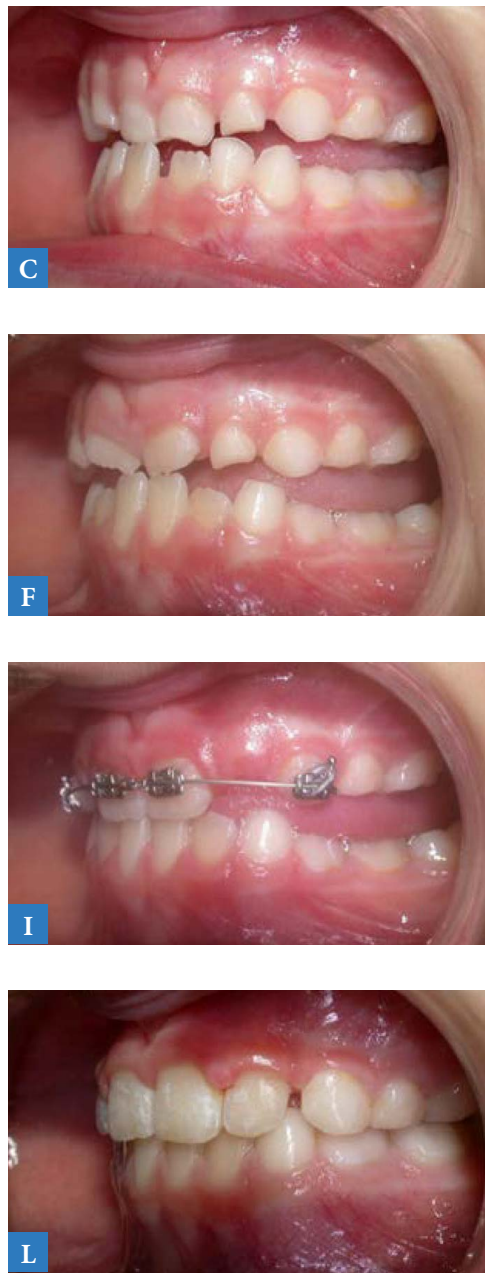

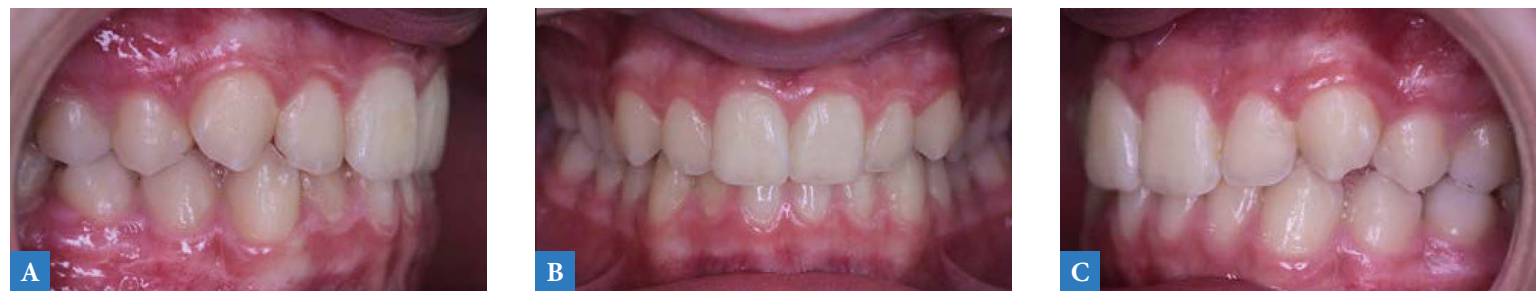

$\triangle$ Fig. 22A à C.

La patiente est revue en denture définitive pour une finition multi-attache qui améliorera l’alignement dentaire.
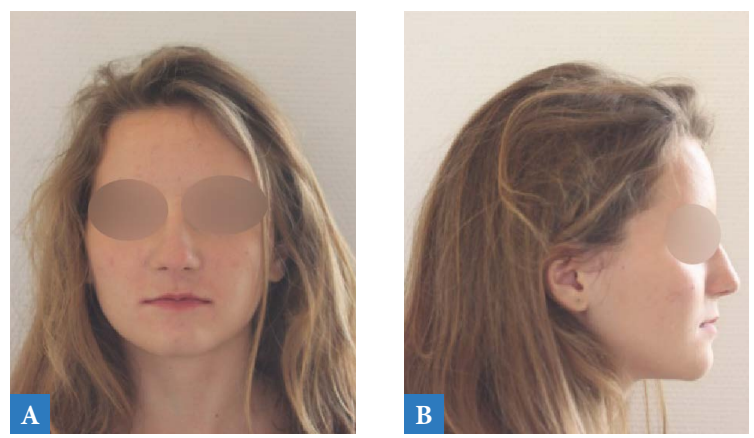

Voici la patiente à 14 ans en fin de traitement multi-attache avec ses radiographies, son visage et son profil sont harmonieux, elle sourit en découvrant ses incisives maxillaires.

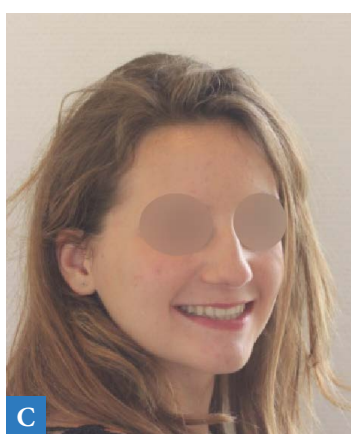

$\triangle$ Fig. 23A à C.
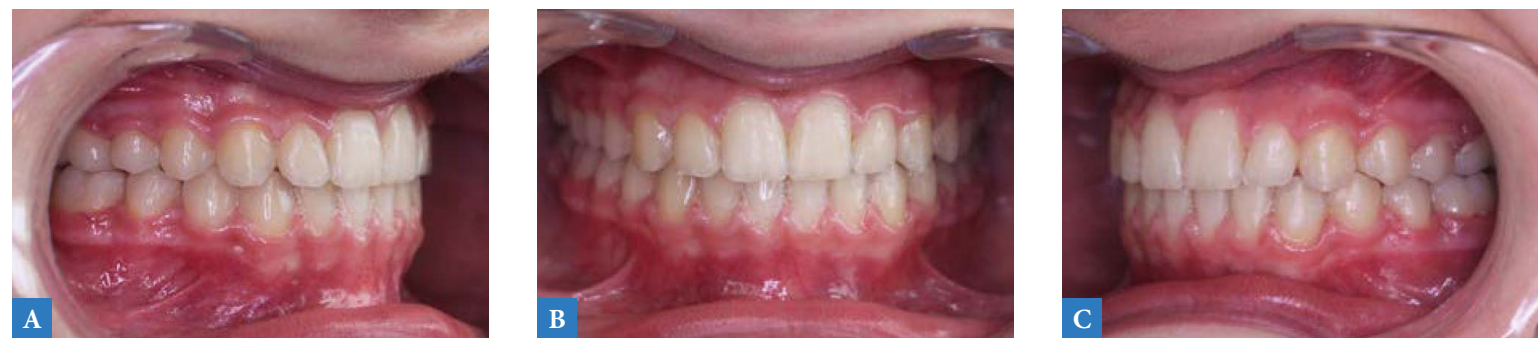

Fig. 24A à C.
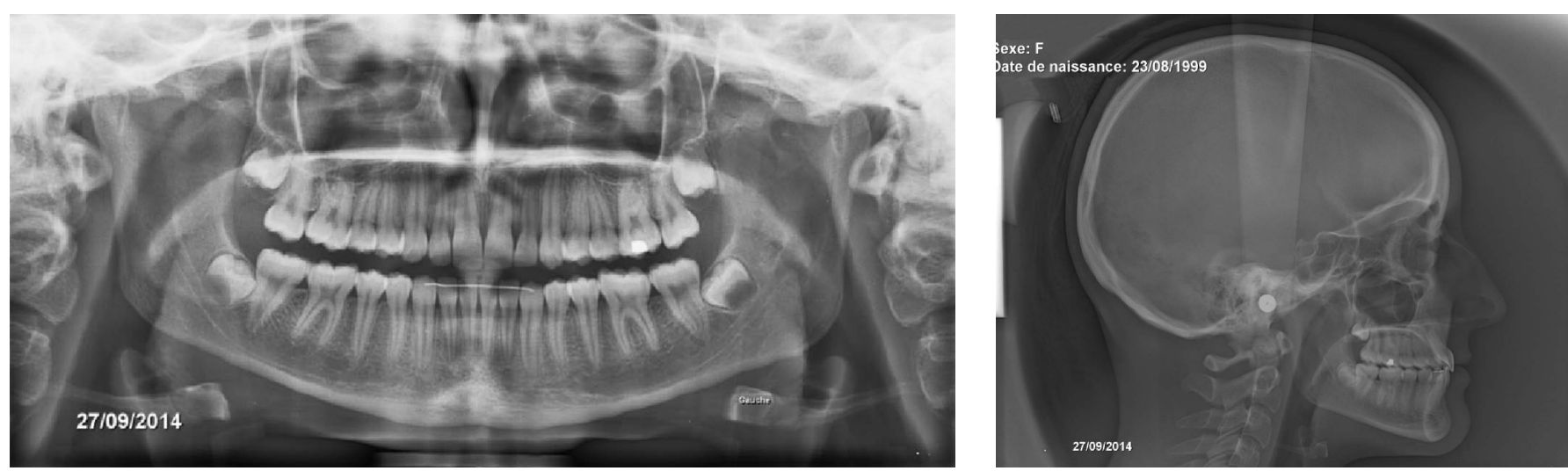

$\triangle$ Fig. 25.

$\triangle$ Fig. 26. 


\section{Cas clinique $n^{\circ} 3$ (Fig. 27 à 36)}

Ce patient de 8 ans, 8 mois présente une classe III avec insuffisance maxillaire et prognathie mandibulaire. L'angle naso-labial est très ouvert et le patient refuse de sourire.

Il a un articulé incisif inversé et un recouvrement incisif, le centre inter-incisif inférieur est dévié vers l'avant et la gauche.

La 12 n'a pas de place pour faire son éruption, mais les incisives inférieures ont des diastèmes.

Le panoramique confirme le manque de place au maxillaire. Dans tous ces cas, l'encombrement dentaire concerne surtout l'arcade supérieure, et témoigne de l'hypoplasie; avec la reprise de croissance de cette arcade, le manque de place s'amendera spontanément. Les extractions monomaxillaires sont à proscrire, elles ne feraient qu'aggraver le déficit squelettique par un déficit alvéolaire.
La téléradiographie montre la grande taille mandibulaire et le retrait maxillaire dont la croissance antéro-postérieure est bloquée.

Le traitement a débuté par une surélévation molaire, les activations du vérin médian stimulent le maxillaire, permettant une croissance homothétique transversale et antéro-postérieure.

La croissance maxillaire crée la place pour toutes les incisives (Fig. 31A à C).

Les incisives mandibulaires sont reculées, grâce aux attaches inférieures (Fig. 32A à C).

Des attaches sur les incisives maxillaires permettent leur alignement (Fig. 33A à C).

En même temps que le rétablissement de l'occlusion incisive, le relief de l'étage moyen de la face change. La lèvre supérieure retrouve un volume harmonieux, l'angle naso-labial se referme et les pommettes commencent à se dessiner, plus rien dans son visage ne trahit l'ancienne classe III (Fig. 34A à C).
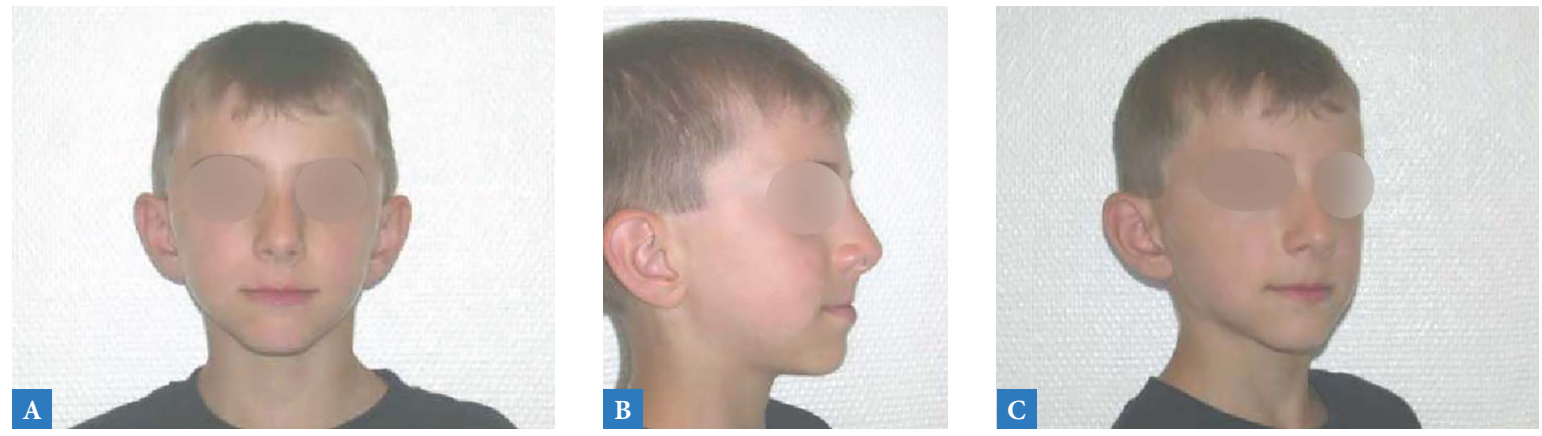

$\triangle$ Fig. 27A à C.
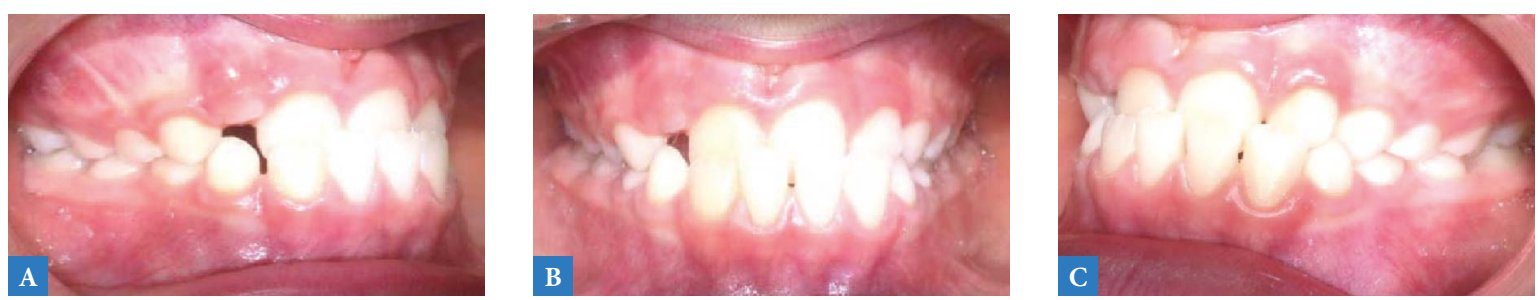

$\triangle$ Fig. 28A à C.

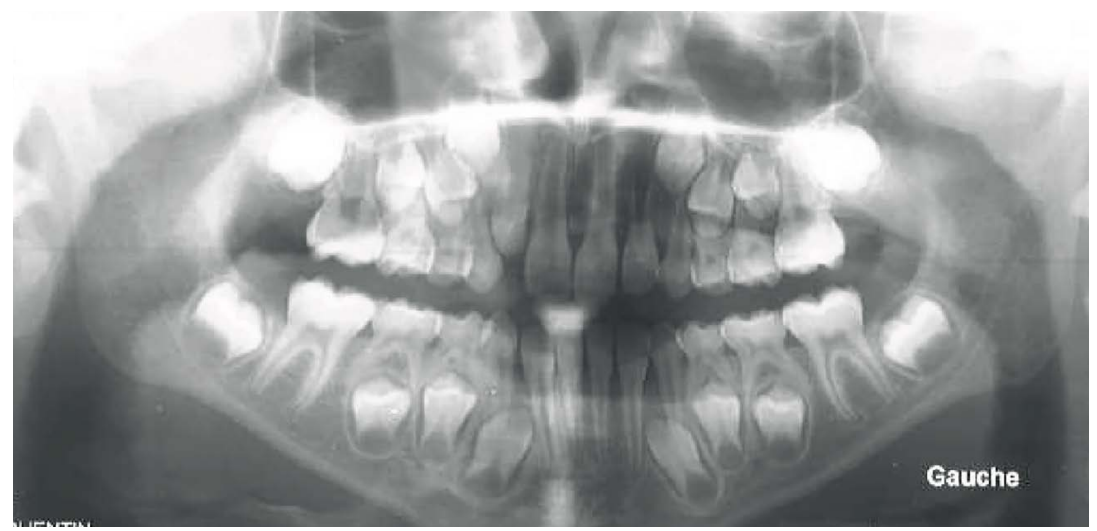

ㄱig. 29.

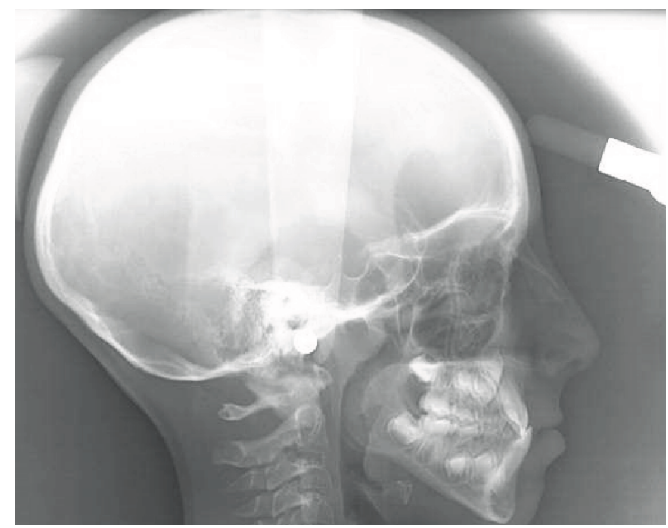

Fig. 30. 

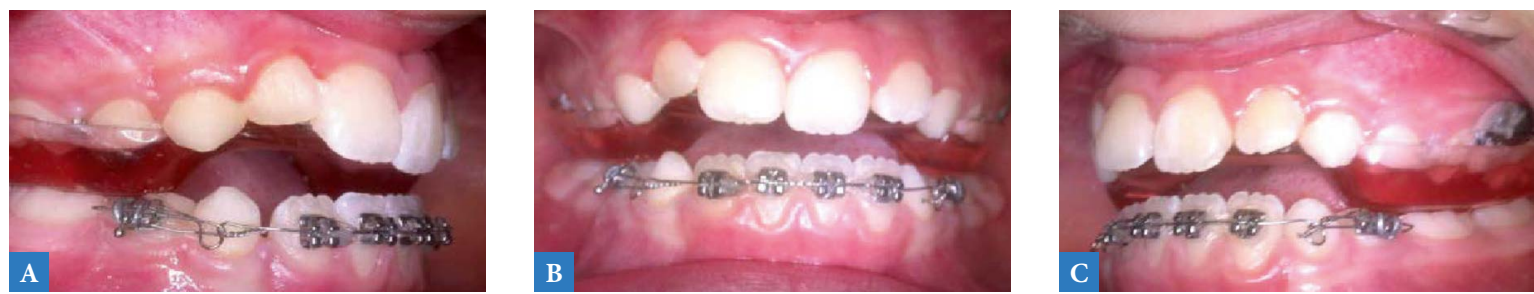

$\triangle$ Fig. 31A à C.
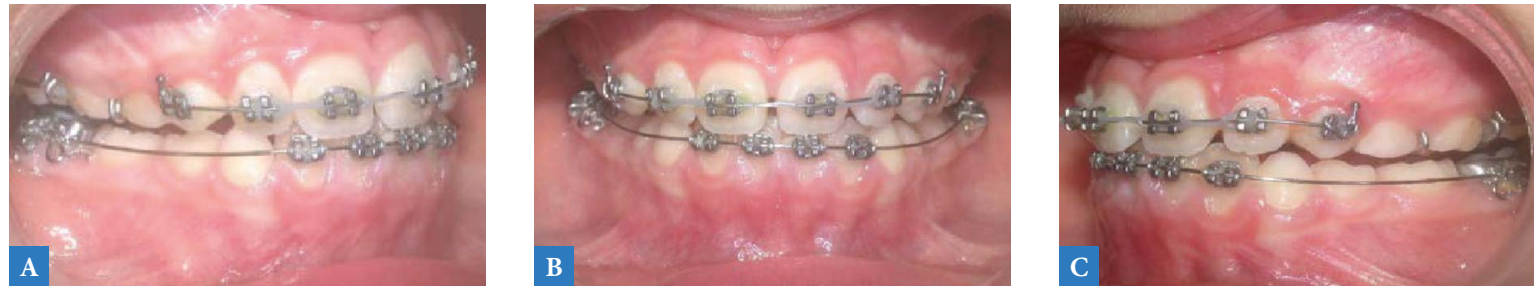

$\triangle$ Fig. 32A à C.
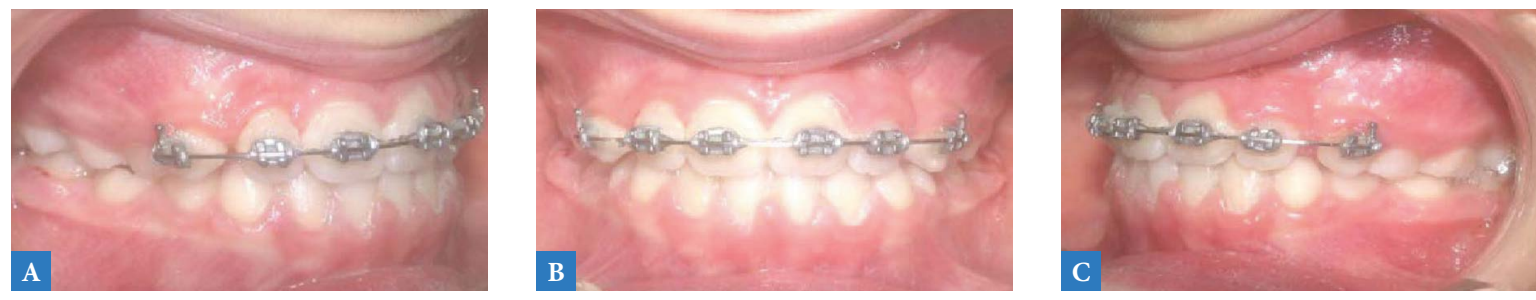

$\triangle$ Fig. 33A à C.
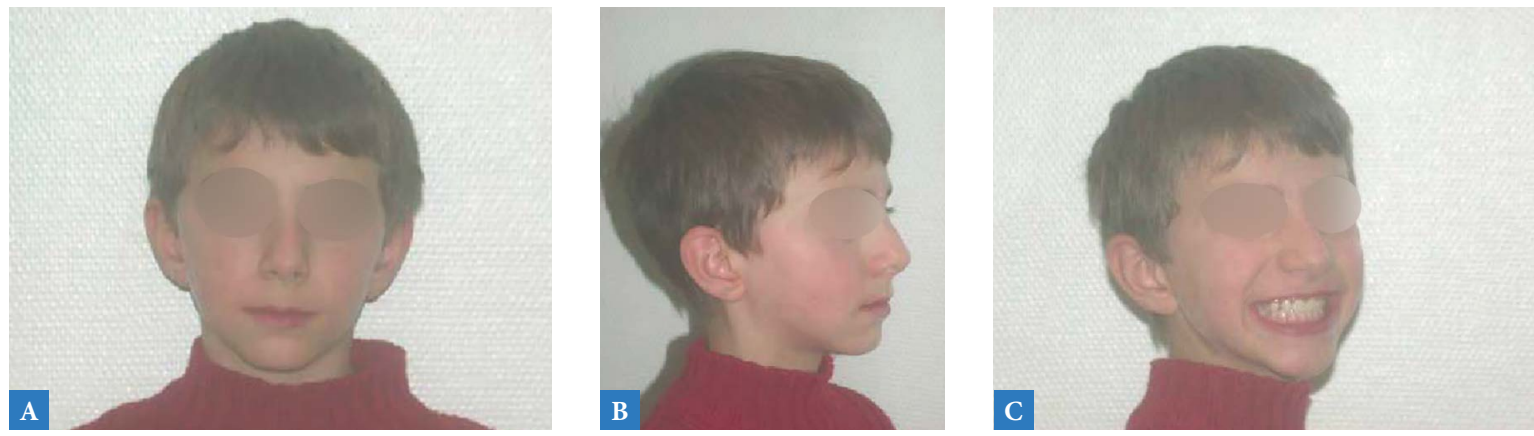

Fig. 34A à C.

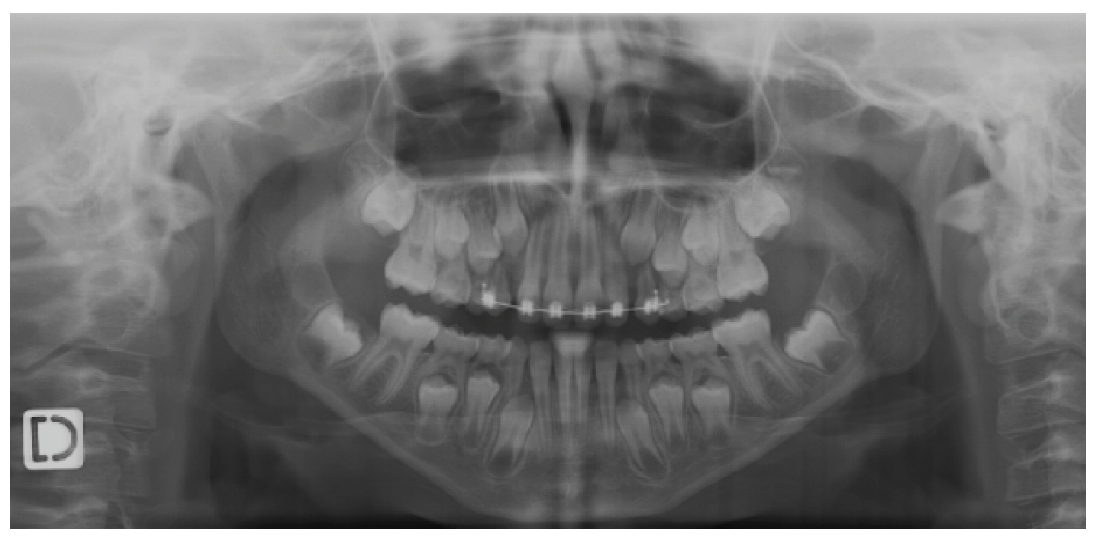

$\triangle$ Fig. 35.

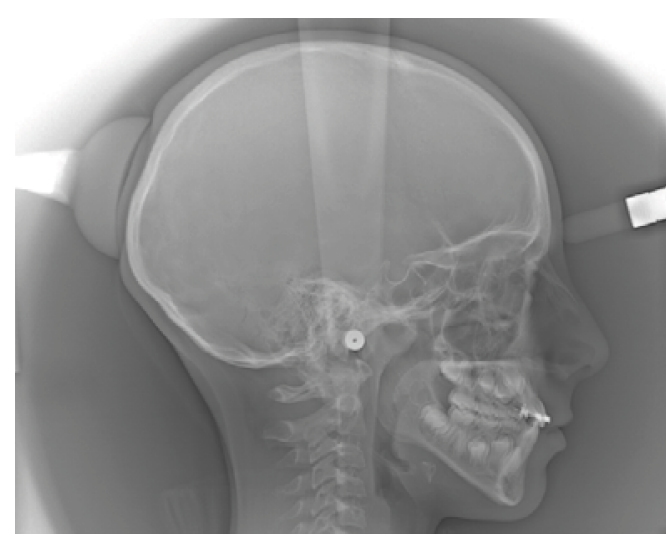

$\triangle$ Fig. 36. 
Cas clinique $n^{\circ} 4$ (Fig. 37 à 44)

Même sans prise en charge des dents mandibulaires, la surélévation seule promeut la croissance verticale antérieure des maxillaires et ferme l'occlusion [12].
Dans le cas ci-dessous on obtient une fermeture de la béance sans aucun appareillage supplémentaire à l'arcade inférieure.
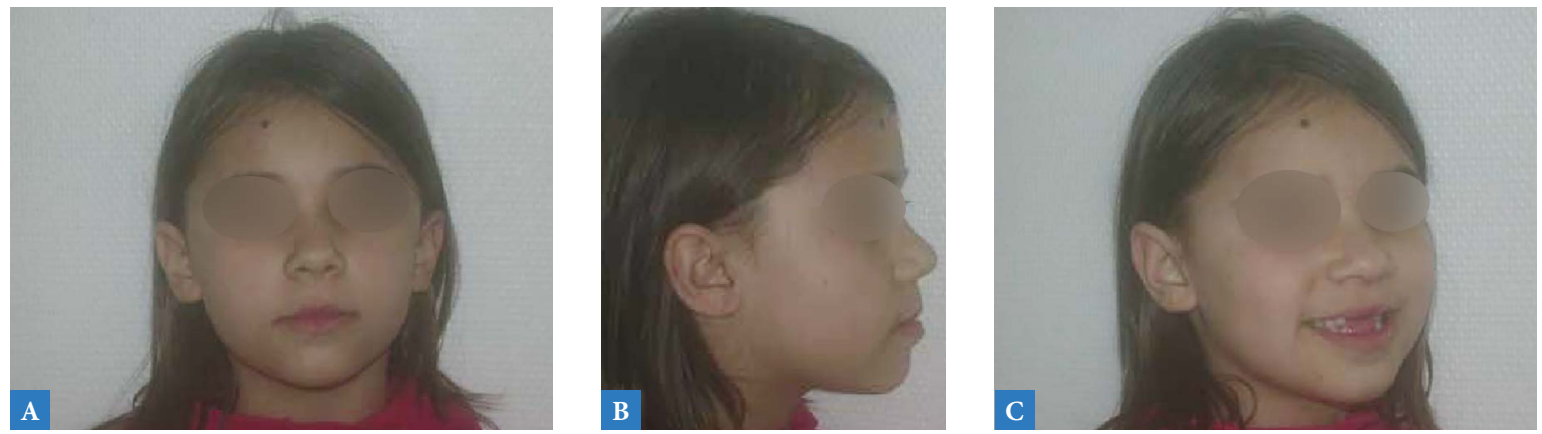

$\triangle$ Fig. 37A à C.
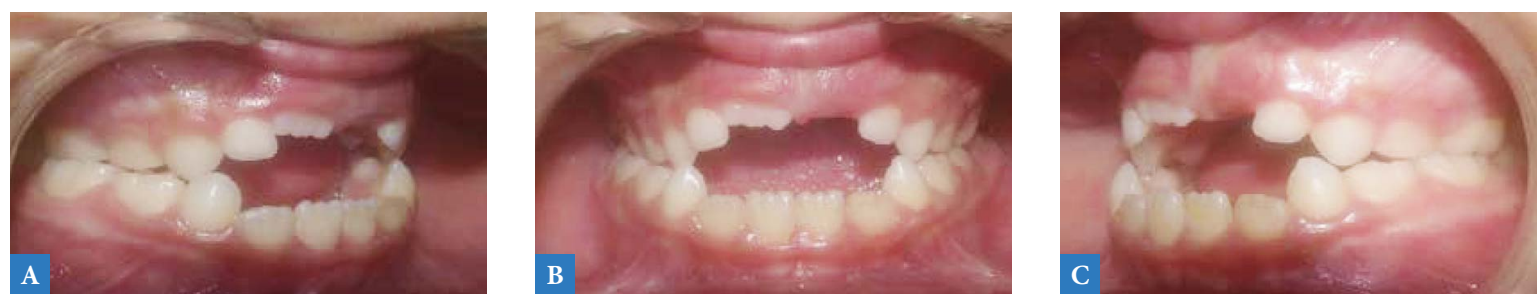

$\triangle$ Fig. 38A à C.

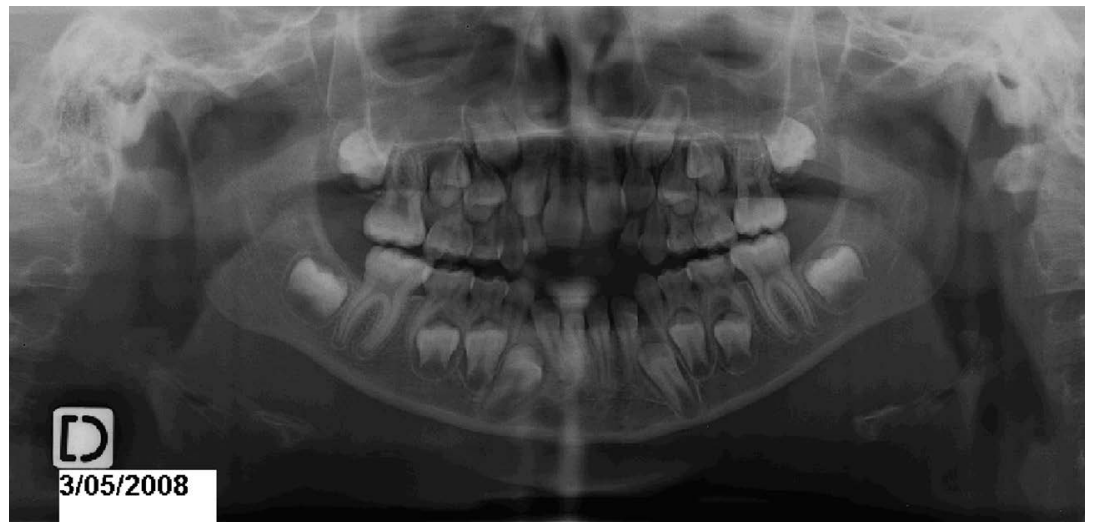

Aig. 39.

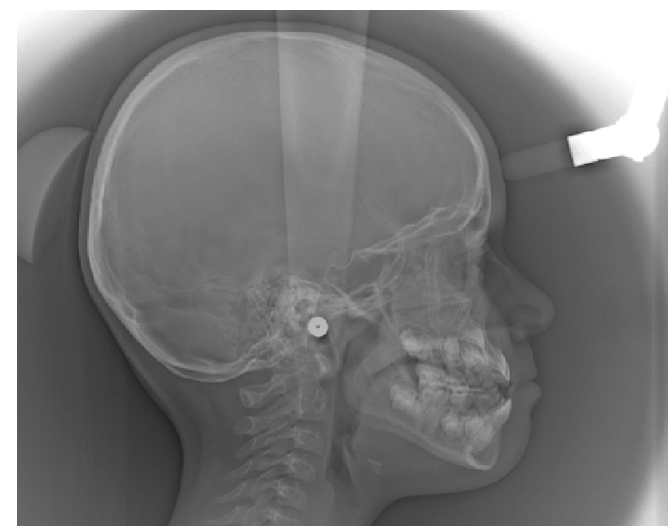

$\triangle$ Fig. 40.
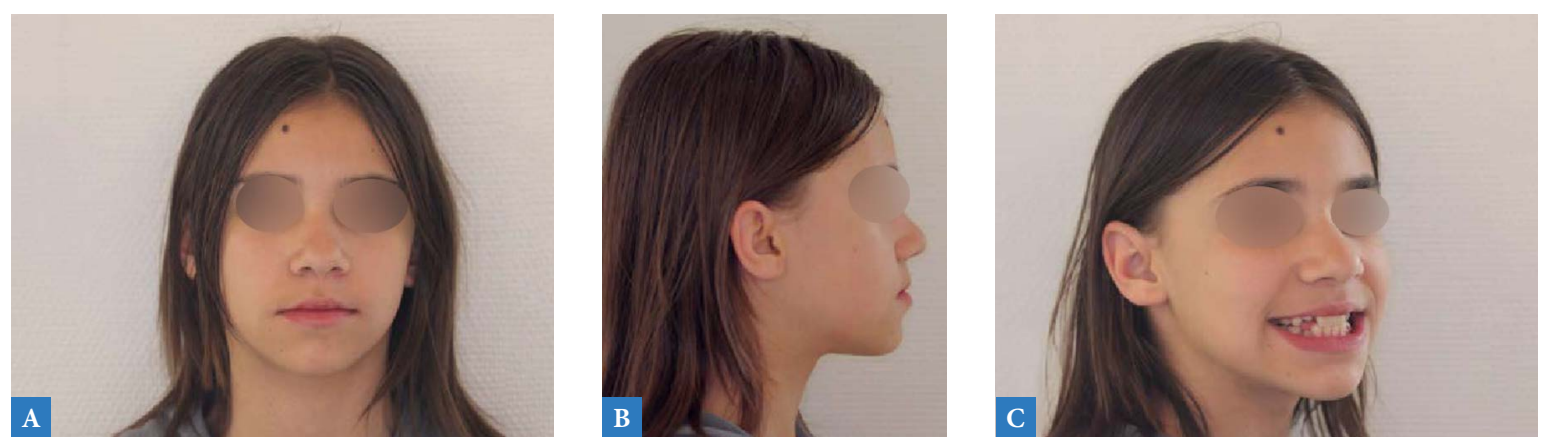

Fig. 41A à C.
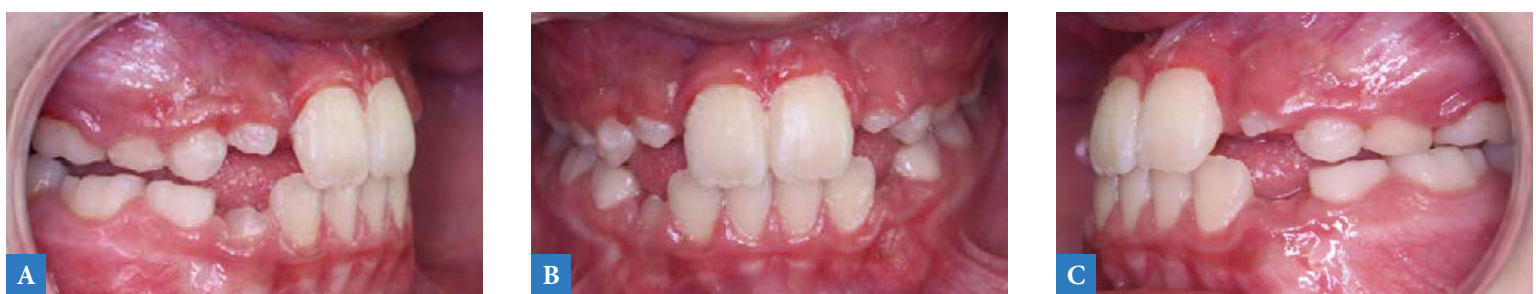

Fig. 42A à C. 


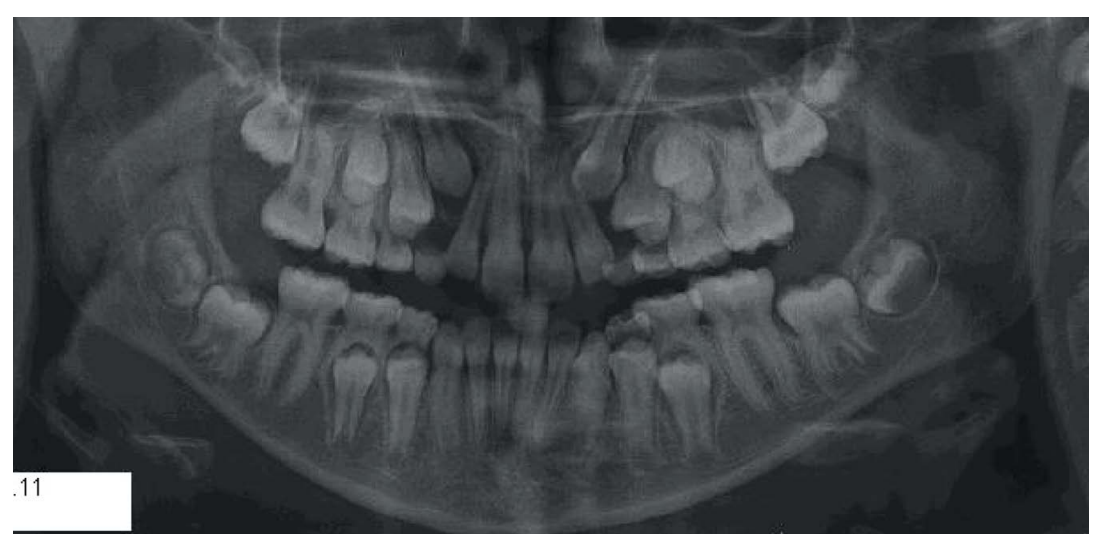

Fig. 43.

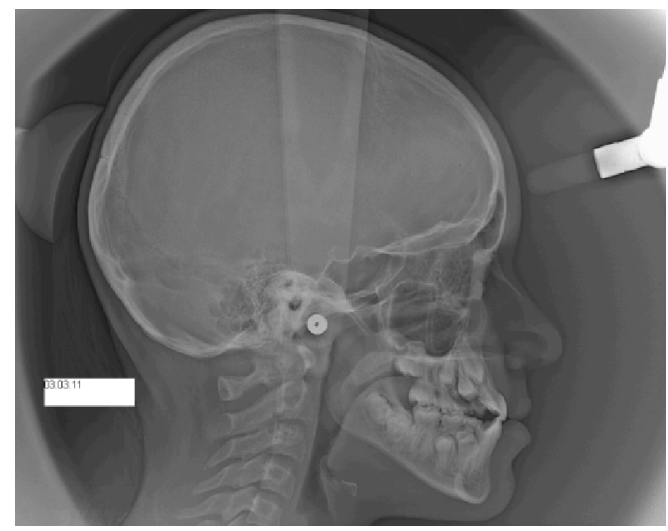

$\triangle$ Fig. 44.

\section{Cas clinique $n^{\circ} 5$ (Fig. 45 à 55)}

Les schémas faciaux hyperdivergents sont les plus difficiles à contrôler et à stabiliser car des difficultés fonctionnelles y sont souvent présentes : respiration nasale déficiente, difficile à maîtriser parce que peu de notre ressort, mauvaise posture linguale, déglutition primaire persistante, interposition linguale à la phonation... Dans ces cas obtenir un recouvrement incisif est essentiel à la pérennité des résultats.
Le mouvement de bascule de l'arcade mandibulaire pour aider la fermeture de la béance va dans le sens de la compensation de la classe III, mais le mouvement induit par l'appareil à plans molaires à l'arcade maxillaire est dans le sens horaire, contraire aux compensations, il a une action réellement orthopédique. Les rapports canins sont de plus en plus en classe III au fur et à mesure que le contact inter-incisif s'établit.
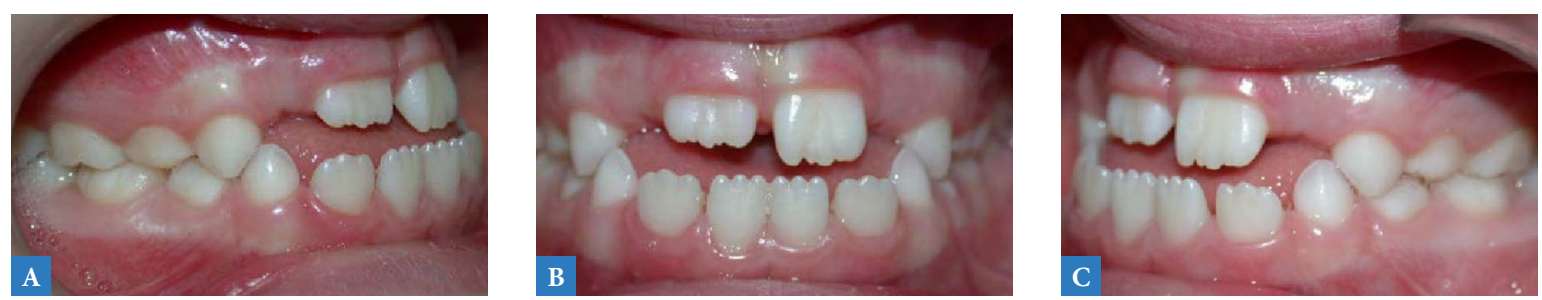

$\triangle$ Fig. 45A à C.

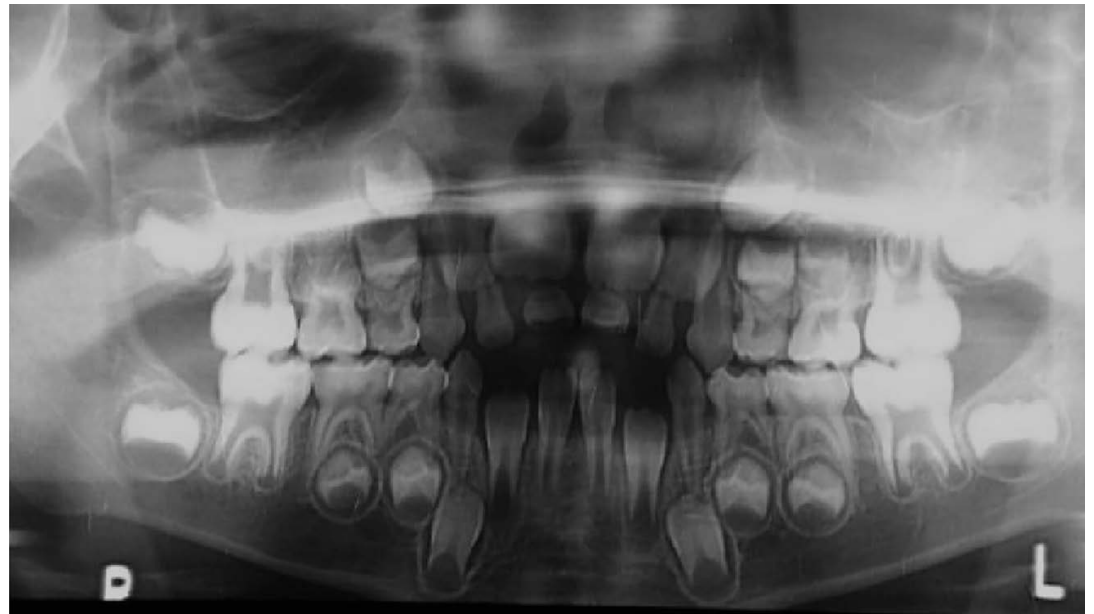

$\triangle$ Fig. 46.

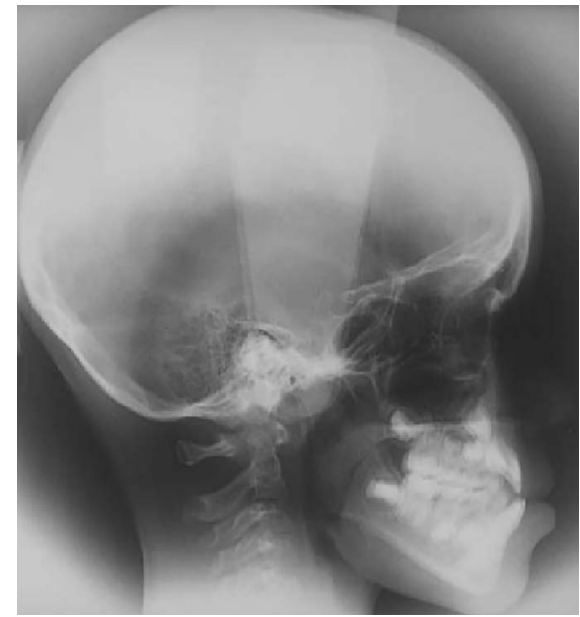

Fig. 47. 

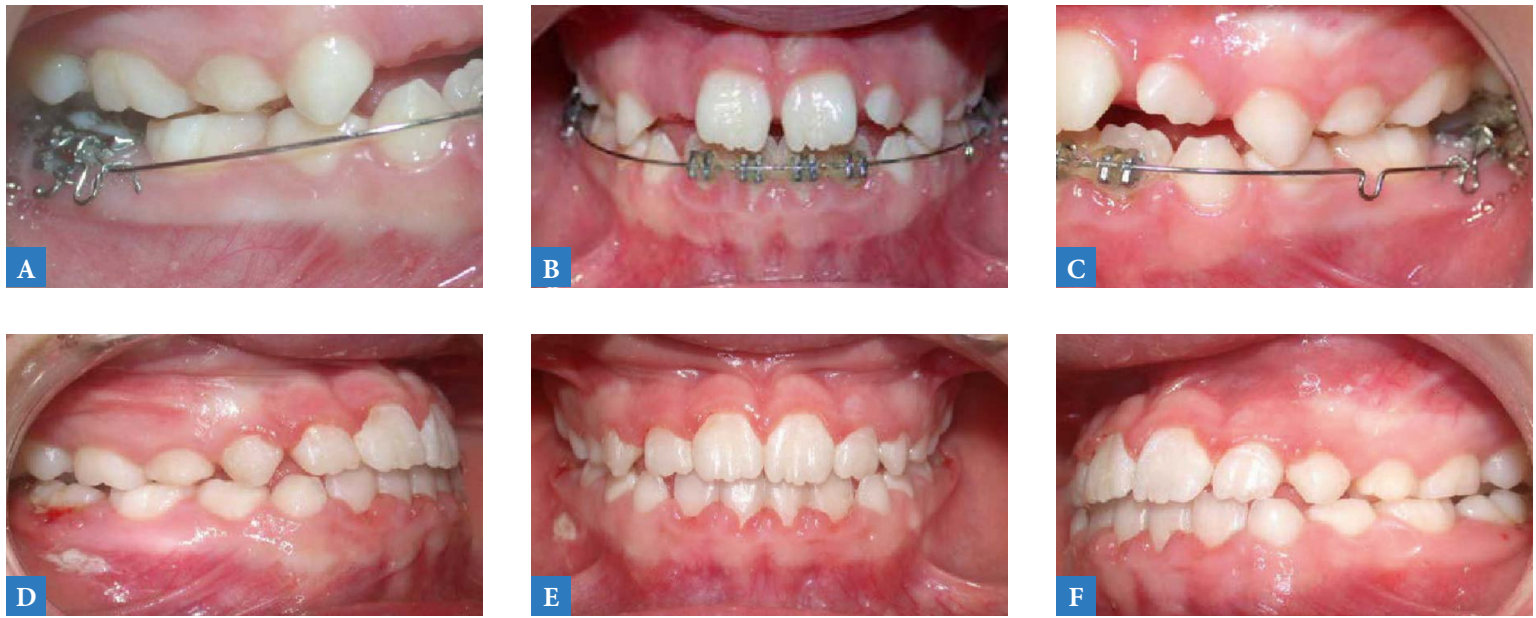

$\triangle$ Fig. $48 \mathrm{~A}$ à $\mathrm{F}$.
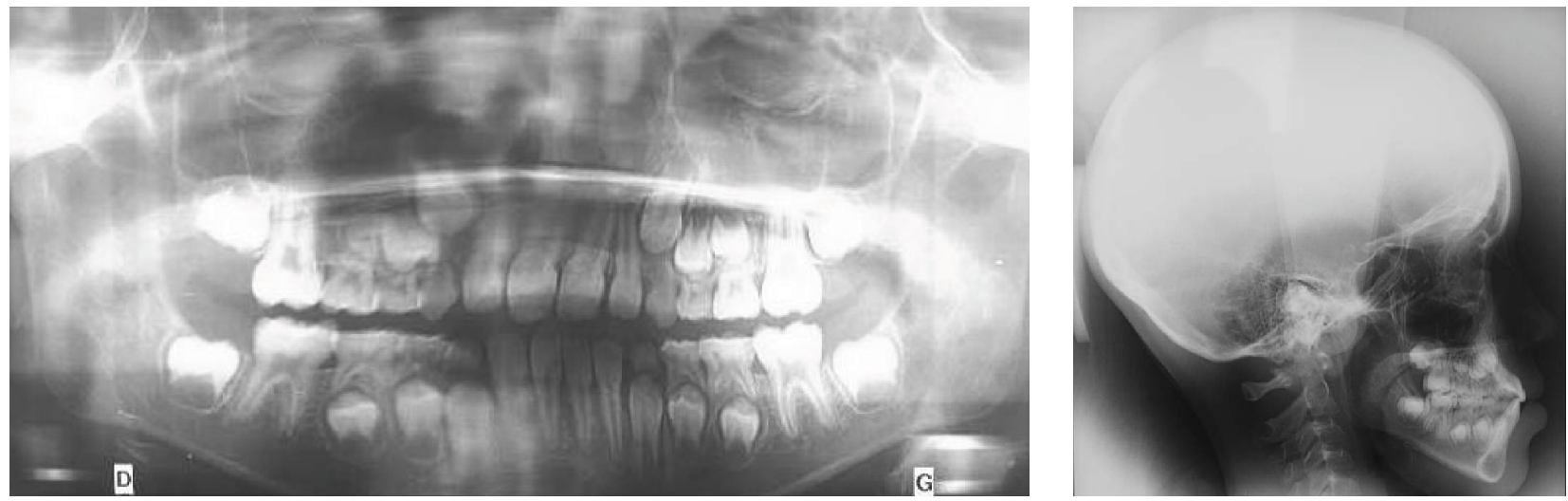

$\triangle$ Fig. 49.

$\triangle$ Fig. 50.
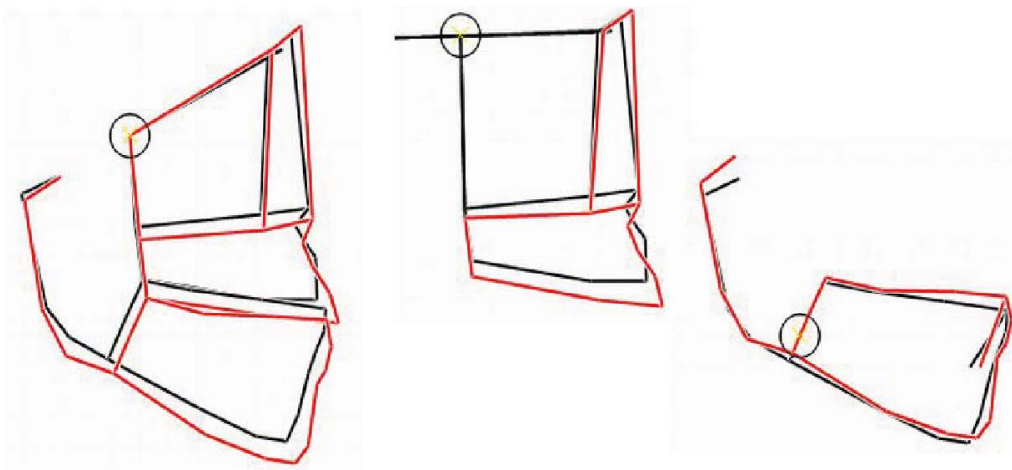

$\triangle$ Fig. 51.

L'étude des superpositions confirme que l'on a bien eu une descente et une avancée du maxillaire, témoignant de la croissance verticale et antéro-postérieure de l'étage moyen de la face, tandis que l'on a une rotation antérieure de la mandibule, pour un schéma facial de départ hyperdivergent, en rotation postérieure.

Après plusieurs années d'interruption, la patiente est baguée à l'arcade mandibulaire uniquement pour utiliser le E-space et reculer les dents antérieures.
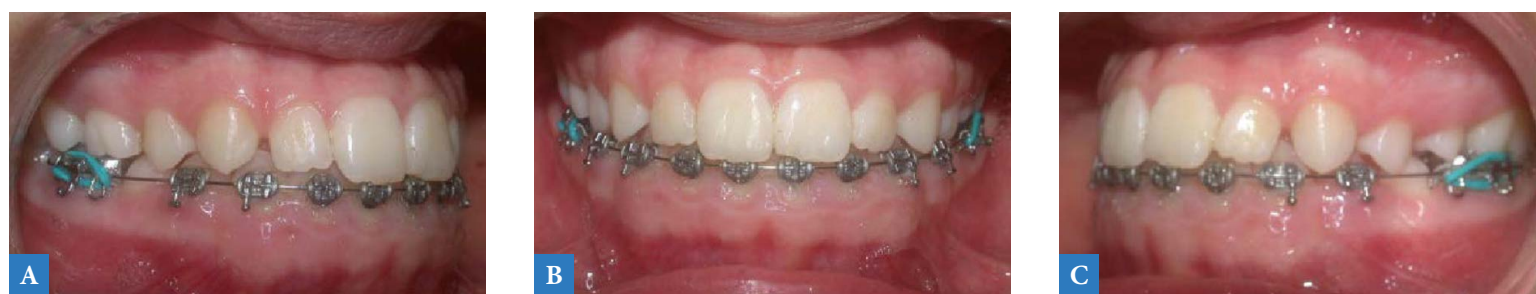


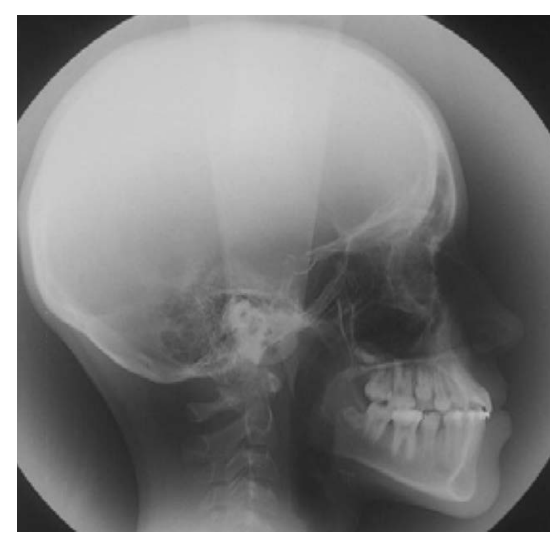

Fig. 53.

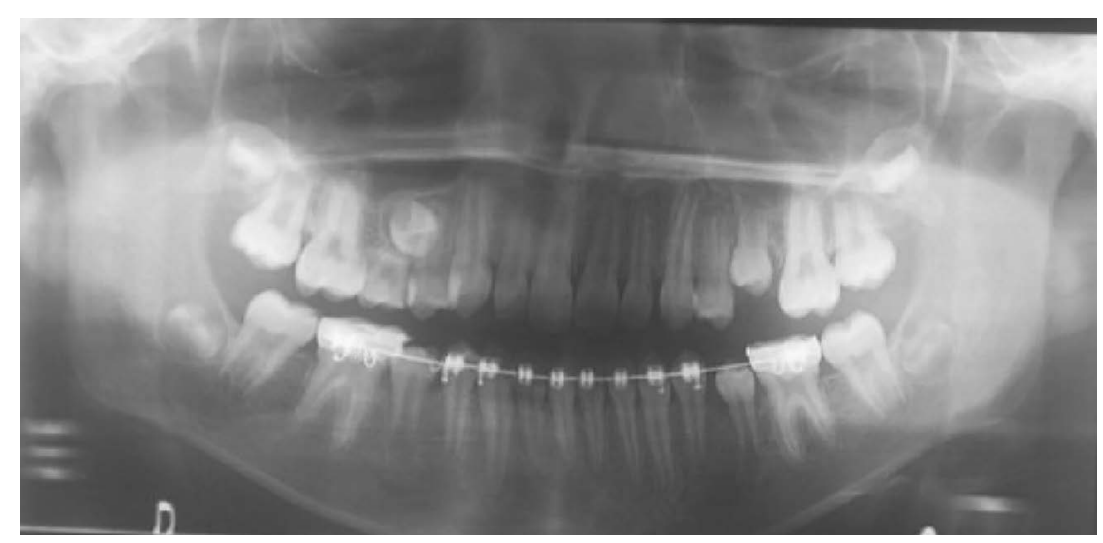

Fig. 54.

4 ans après la dépose, un élastique en criss/cross remettra la 15 qui est apparue tardivement sur la
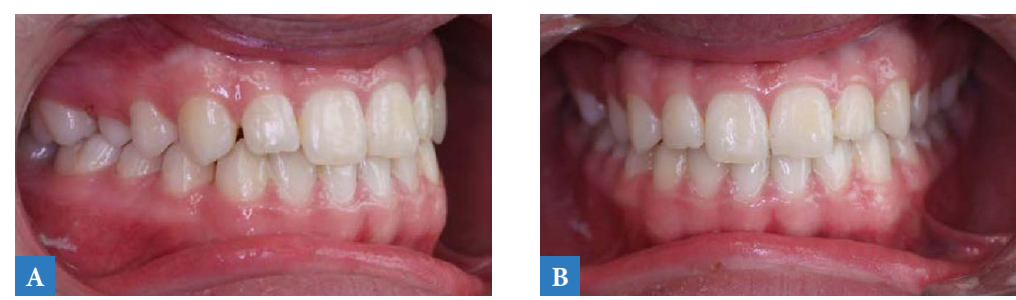

$\triangle$ Fig. 55A à C.

Cas clinique $n^{\circ} 6$ (Fig. 56 à 63)

Il est préférable, plus simple et plus rapide de traiter des enfants avant la puberté, mais quand ils viennent tardivement, une prise en charge est réalisable.
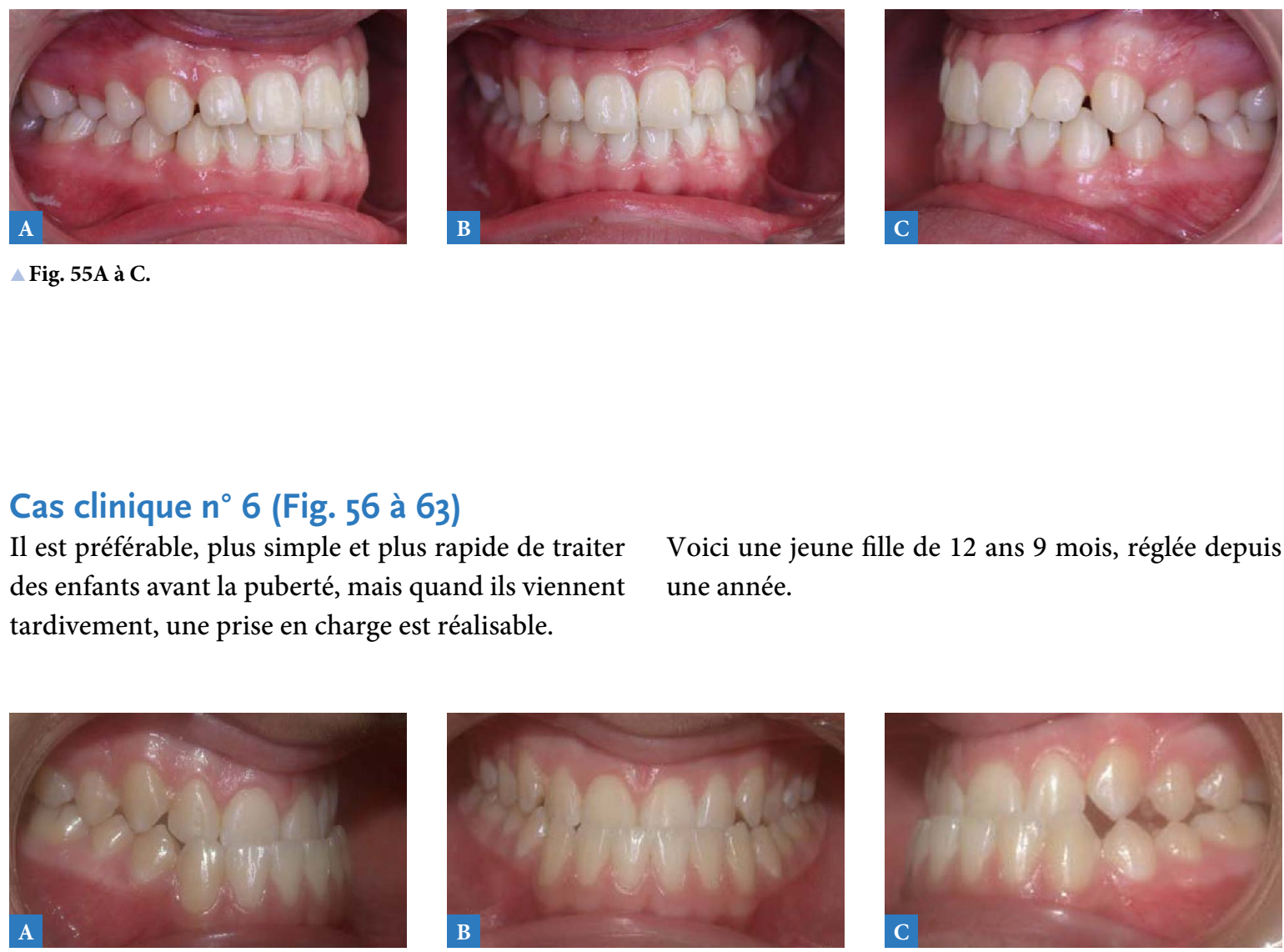

panoramique et ayant un retard de maturation, fera une éruption extrêmement tardive en position palatine.

Voici une jeune fille de 12 ans 9 mois, réglée depuis une année.

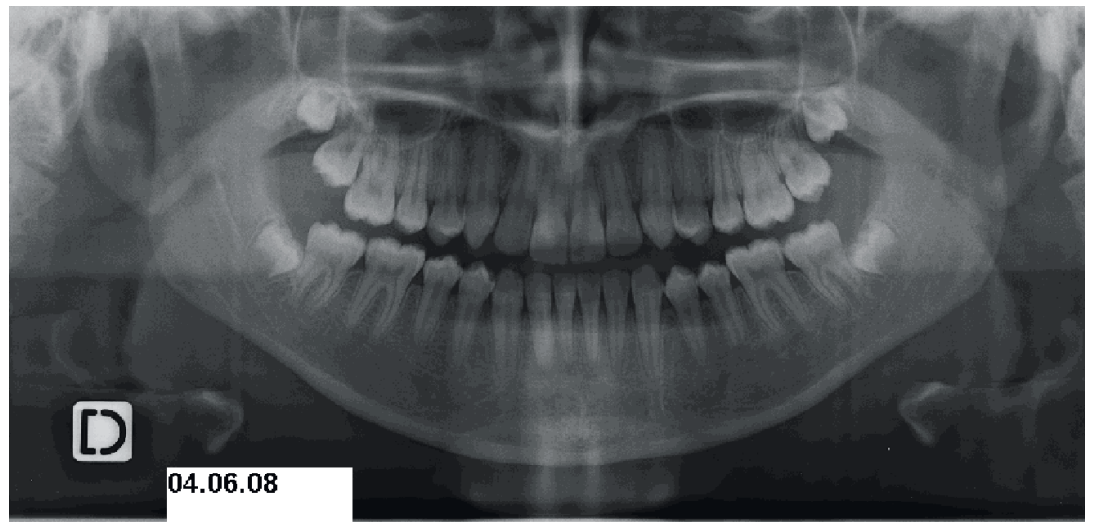

Fig. 57.

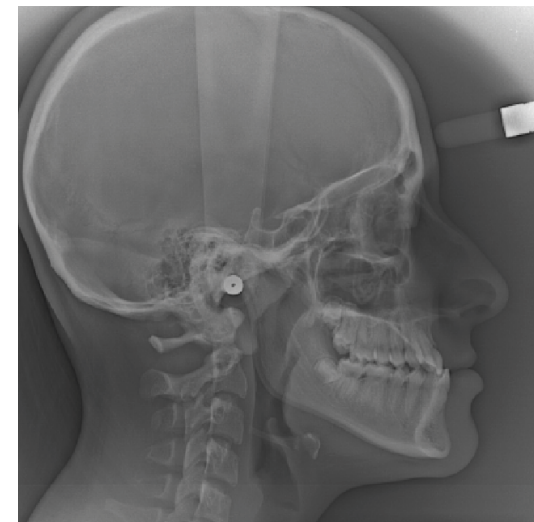

Fig. 58. 
Nous avons d'abord mis en place une surélévation, puis appareillé l'arcade mandibulaire, afin d'utiliser une force directionnelle pour maintenir les dents inférieures, on pourrait aussi imaginer d'utiliser des mini-vis. Le maxillaire est bagué quand le surplomb incisif correct est obtenu.

Les dents antérieures sont munies des brackets hypertorqués à l'arcade inférieure et hypotorqués à l'arcade supérieure, les fils rectangulaires ne doivent être engagés qu'une fois le recouvrement incisif suffisant, afin que l'occlusion maintienne les couronnes pour obtenir le déplacement des racines. Le torque positif sur les incisives mandibulaires permet de creuser le sillon labio-mentonnier.
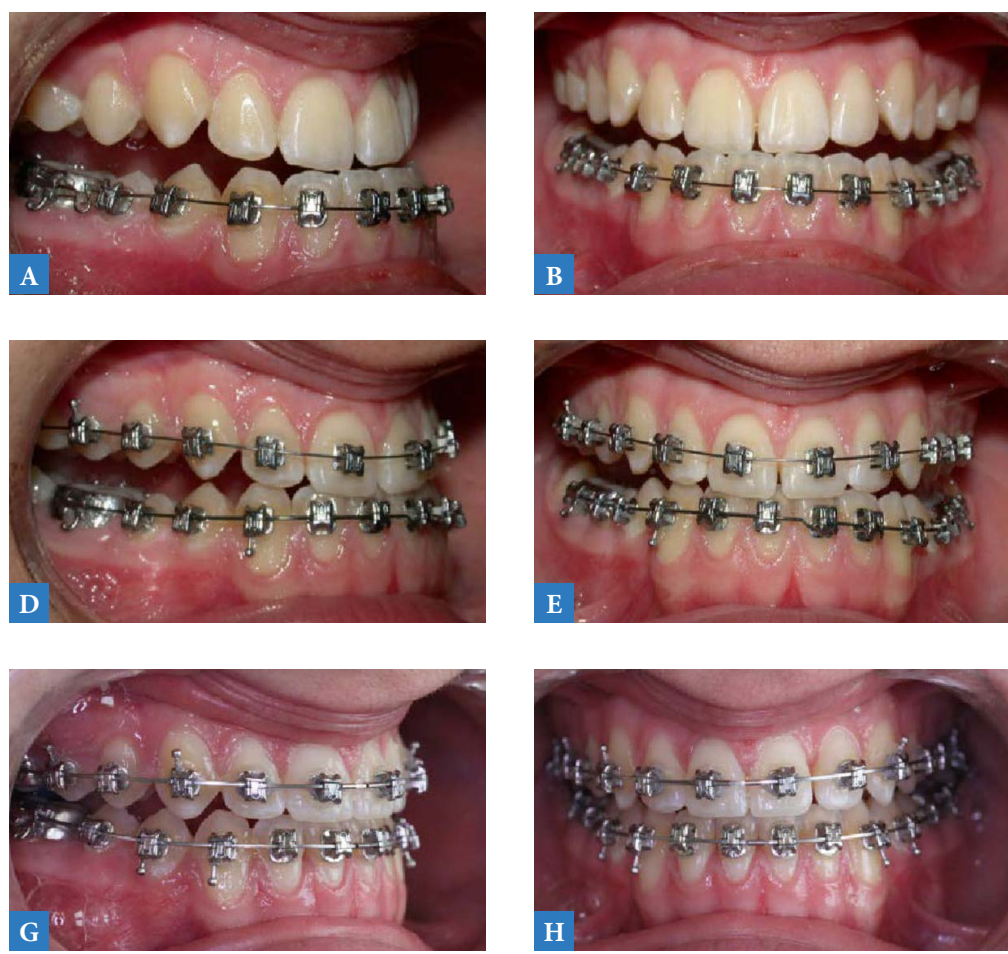

$\triangle$ Fig. 59A à I.
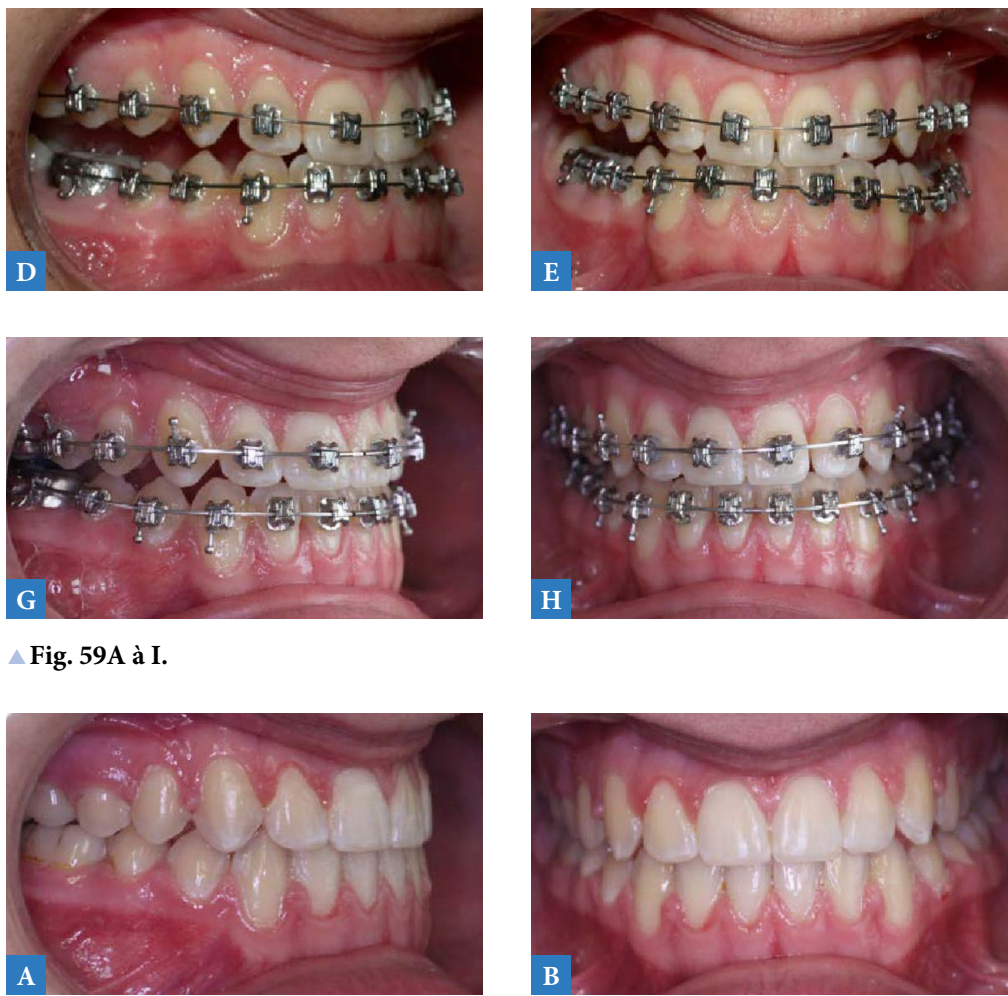

$\triangle$ Fig. 60A à C.
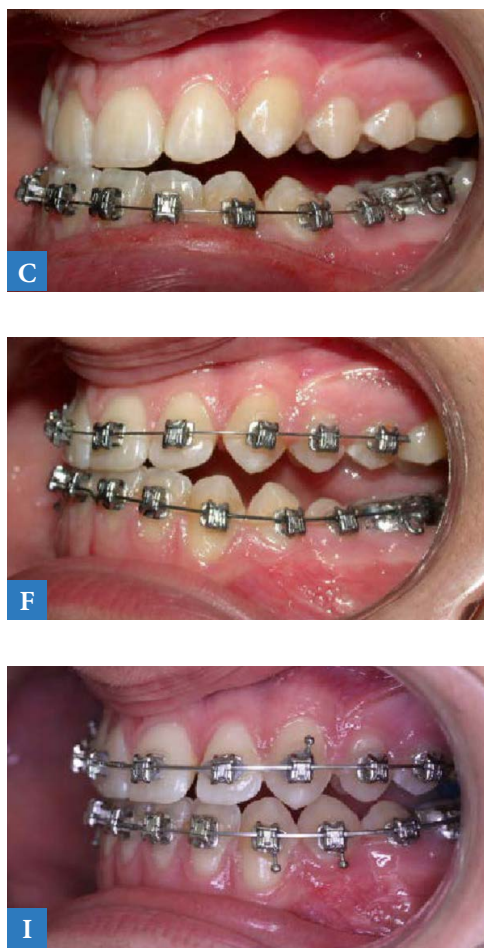

F
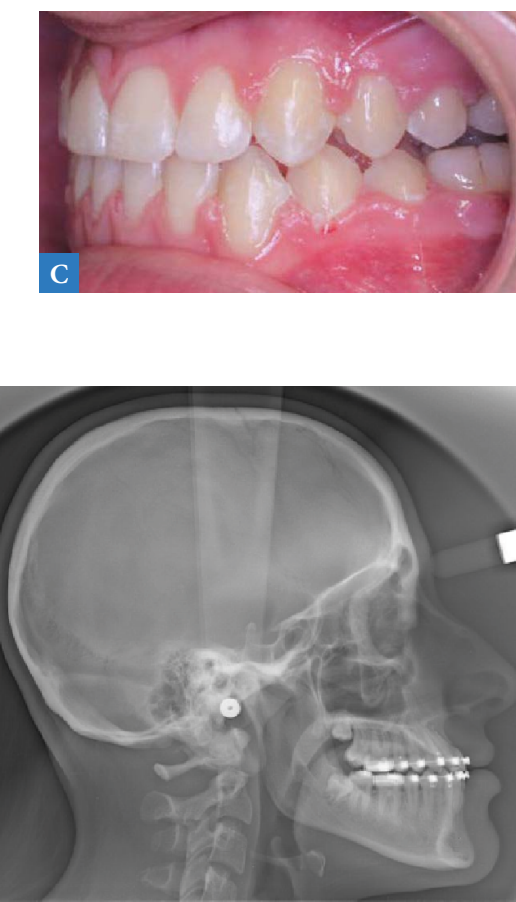

$\triangle$ Fig. 62.

$\triangle$ Fig. 61. 
Les superpositions obtenues avec une radiographie réalisée en cours de traitement montrent la croissance obtenue au maxillaire.
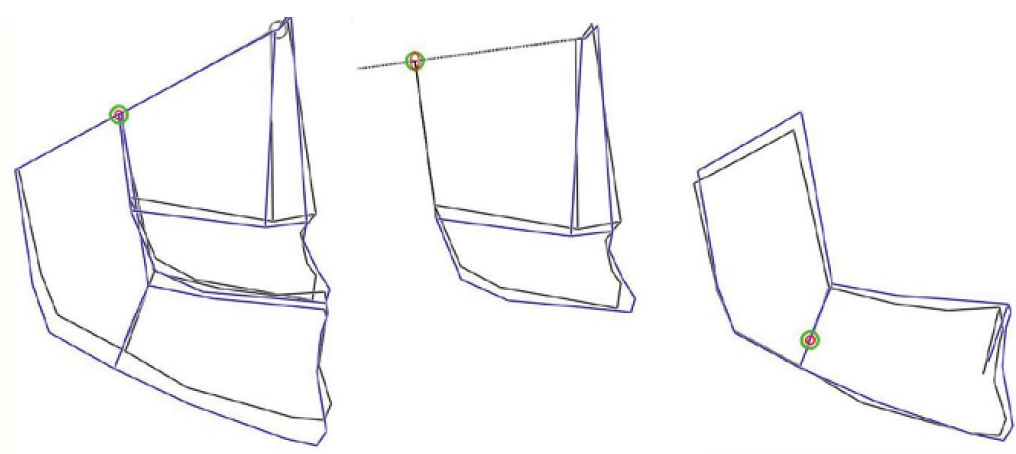

Fig. 63.

\section{LA CONTENTION}

La rééducation orthophonique est un excellent moyen de contention en jugulant les dyspostures et les dysfonctions linguales. Mais l'appareil en soi est un système de rééducation des fonctions : en élargissant le palais on élargit la base des fosses nasales, on améliore la respiration. À l'issue du traitement, on a constaté une élévation de l'os hyoïde signant l'ascension de la base linguale.

L'ostéopathie limite les blocages et encourage la mobilité des structures osseuses, ce qui va dans le sens de nos traitements.

Parmi les différents systèmes de contention nous avons utilisé: l'activateur de classe III à port nocturne avec un bandeau d'Eschler partant de la plaque maxillaire pour recouvrir les incisives mandibulaires, l'allongement des incisives maxillaires au composite qui est l'équivalent du ressort d'Eschler fixé et qui majore le recouvrement incisif obtenu, le maintien des incisives inférieures et surtout l'augmentation de la dimension verticale d'occlusion qui peut s'associer ou non aux systèmes précédents.

Une surélévation en composite collée sur les dernières dents en bouche permettra de maintenir de façon non contraignante les résultats acquis. Les dents lactéales peuvent être reconstituées en composite pour augmenter leur hauteur. Quand nous avons la chance de traiter les enfants tôt, la contention la plus simple est la surélévation au composite des secondes molaires de lait au niveau du contact 55-85 et 65-75, permettant de majorer l'éruption des 6 et d'augmenter de façon stable la DVO, cette contention s'éliminera à la chute des dents de lait et si elle est faite au composite de couleur, peut être observée quand à son devenir.

Dans les cas de délabrement des molaires, fréquent dans les MIH, la reconstruction par coiffes pédodontiques consolidera solidement l'avancée maxillaire.

\section{CONCLUSION}

Les 3 sens de l'espace sont interdépendants, en agissant sur le sens vertical et sur le sens transversal, on agit sur le sens antéro-postérieur, et vice versa. L'étude de ces cas de prognathie mandibulaire et d'hypoplasie maxillaire traités par surélévation molaire associée ou non aux autres systèmes orthodontiques, donne des résultats satisfaisants. Le dispositif amovible et simple remplace les masques faciaux très encombrants et évite les interventions chirurgicales mutilantes. Il permet une prise en charge précoce dont les conséquences dépassent le cadre de l'occlusion et de la fonction dentaire. On a dès le jeune âge une amélioration globale de l'esthétique faciale ayant des répercussions positives sur la psychologie et l'image de soi du petit patient. Mais l'augmentation de la dimension verticale d'occlusion donne aussi la possibilité de traitements plus tardifs. 


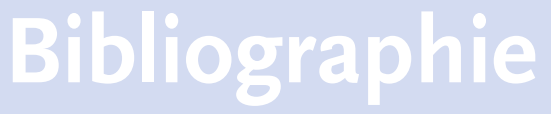

[1] Miyajima K, McNamara JA Jr, Sana M, Murata S. An estimation of craniofacial growth in the untreated Class I female with anterior crossbite. Am J Orthod Dentofacial Orthop 1997; 112(4): 425-34.

[2] Deffez JP. Prognathies mandibulaires. Propositions thérapeutiques. Julien Prélat édit, Paris, 1971.

[3] Deffez JP. Bases de l'étage moyen de la face. Les sites et les facteurs de croissance du maxillaire : données actuelles. Actual OdontoStomatol 1979; 128: 647-68.
[4] Deffez JP, Fellus P, Franchi-Deffez I, Huard JL. Approche comportementale des troubles de l'articulé dentaire. Rev Int Pédiatr 1987; 174: 5-20.

[5] Lepoivre M, Garlopeau F, Laudenbach P. Prognathisme mandibulaire traité par surélévation temporaire de l'articulé. Actual Odonto-Stomatol 1959; 47: 577-290.

[6] Fellus P. Orthodontie précoce en denture temporaire. Cah Proth édit, Paris, 2003.

[7] Couly G. La langue, appareil naturel d'orthopédie dento-faciale pour le meilleur et pour le pire. Rev Orthop Dento-Faciale 1989; 23: 9-17.

[8] Ben Younes-Uzan C. Proposition non conventionnelle de traitement des classes III. Orthodont Française 2007; vol 82(2):133-9.
[9] Delaire J. Architectonique et dynamique cranio-faciale. Cours d'enseignement postuniversitaire de stomatologie pédiatrique. Hôpital Robert Debré, 1992-1993.

[10] Delaire J, Verdon P, Salagnac JM, Felpetto $\mathrm{Y}$, Zayat $\mathrm{S}$. Bases physiologiques de l'équilibre du maxillaire supérieur. Incidences en ce qui concerne le mode d'action des forces lourdes extra-orales. Actual. Odonto-Stomatol 1979; 128: 611-645.

[11] Planas $\mathbf{P}$. La réhabilitation neuroocclusale. Masson édit, Paris, 1992.

[12] Ben Younes-Uzan C. Fermeture des infraclusions par mastication sur les dents postérieures. Rev. Orthop. Dento-Faciale 2017; 51: 447-455. 